\title{
Development and Progress in Piezotronics
}

Authors: Xiaonan $\mathrm{Wen}^{\mathrm{a}}$, Wenzhuo $\mathrm{Wu}^{\mathrm{a}}$, Caofeng $\mathrm{Pan}^{\mathrm{b}}$, Youfan $\mathrm{Hu}^{\mathrm{c}}$, Qing Yang ${ }^{\mathrm{d}}$ and Zhong Lin Wang ${ }^{\text {ab* }}$

a School of Materials Science and Engineering, Georgia Institute of Technology, Atlanta, Georgia 30332-0245, USA.

b Beijing Institute of Nanoenergy and Nanosystems, Chinese Academy of Sciences, Beijing, China

c Key Laboratory for the Physics and Chemistry of Nanodevices, and Department of Electronics, Peking University, Beijing 100871, China

d State Key Laboratory of Modern Optical Instrumentation, Department of Optical Engineering, Zhejiang University, Hangzhou 310027, China

*Corresponding Author: Email: zlwang@ gatech.edu; Phone: 404-894-8008

Abstract: The coupling of piezoelectric and semiconducting properties gives rise to the effect of piezotronics, which regulates charge carrier transport via the modulation of energy barriers at contact interfaces. With piezoelectric semiconductors as the building blocks, extensive progress has been made, covering the fundamental physics level, the individual device level as well as the integrated system level, effectively establishing a new field of study. By manipulating interfacial processes incorporating ionic charges, free electrons/holes, photons and chemicals, novel interdisciplinary effects have been studied and reported. This paper aims at reviewing the milestone progress and offering perspectives of this new field of study in applications for multi-functional sensing systems, human-electronics interfacing, MEMS, energy harvesting and so on.

Keywords: piezotronic, piezophototronic, transistor, $\mathrm{ZnO}$, sensing, performance enhancement 


\section{Content:}

1. Introduction

2. Fundamentals of piezotronics

2.1. Piezotronic effect on metal-semiconductor (M-S) contact interface

2.2. Piezotronic effect on semiconductor-semiconductor (S-S) contact interface

2.3. Influence of free carrier screening on the piezotronic effect

3. Variations of single piezotronic transistors

3.1. Choice of channel materials

3.2. P-type vs n-type channel materials

3.3. Nanowire vs thin film vs 2-D channel materials

4. Novel applications of piezotronic devices beyond strain sensors

4.1. Piezotronic logic devices

4.2. Piezotronic programmable resistive memories

5. Coupling of the piezotronic effect with other interfacial effects

5.1. Piezophototronics

5.1.1. Enhanced efficiency in inorganic/organic hybrid LEDs by piezophototronic effect

5.1.2. Enhanced efficiency in $\mathrm{ZnO} / \mathrm{P} 3 \mathrm{HT}$ solar cells by piezophototronic effect

5.2. Enhanced chemical sensing by piezotronics

6. Integration of piezotronic devices towards functional systems

6.1.3D piezotronic transistors matrix for electrical imaging of pressure distribution

6.2. 3D piezophototronic LEDs for electroluminescent imaging of pressure distribution

7. Summary and prospects 


\section{Introduction}

The advancement of electronic technology has brought unprecedented changes to all parts of human society and civilization. While scientists and engineers keep pushing the limits of Moore's law, the development of innovative interfacing technologies is equally important so that we can fully utilize the computational power at hand for diverse and smart tasking. The goal is to make electronic products more interactive with the environment and more integrated with humans [1-5]. To achieve this, an effective perception system is needed so that an electronic device can passively receive orders via multiple media as well as actively make decisions by feeling the surroundings. The ability to sense and react to mechanical stimulations is an indispensable function and thus developing devices that translate mechanical signals into electrical signals is of vital importance.

There are two most widely applied mechanisms for pressure sensing. One is the piezoresistive effect [6-8], which says materials' deformation leads to band structure modulation and thus leads to resistance change. This effect is manifest in semiconductor materials and piezoresistive strain sensors work in a passive mode where their resistance is constantly measured to achieve the monitoring of external force/pressure. The other is the piezoelectric effect [9-11], which says deformation of certain materials along specific directions leads to piezoelectric polarization. This effect is manifest in insulating piezoelectric materials and piezoelectric strain sensors work in an active mode where they provide a voltage signal in response to applied force/pressure. The above two types of strain sensors each have their distinct pros and cons: piezoresistive strain sensors have relatively low sensitivity and on/off ratios but could be easily integrated into electronic circuits due to its passive working mode; piezoelectric strain sensors have high sensitivity and accuracy by actively outputting voltage signals but do not directly fit into electronic circuits.

A third strain sensing mechanism, called the piezotronic effect, has emerged in recent years, smartly hybridizing the advantages of the above two and has shown very bright prospects as the next generation pressure sensing and electronic interfacing technology [12-14]. This effect utilizes the direct coupling between piezoelectricity and electronics, which raises two requirements for the functioning material: being piezoelectric and being semiconducting. A number of II - VI and III - V semiconductors such as $\mathrm{ZnO}, \mathrm{GaN}$ and $\mathrm{CdS}$ fall into this category. Although not having as large piezoelectric coefficients, the semiconducting nature of these materials enables effective tuning of surface/interfacial energies by strain induced piezoelectric potential and thus the corresponding modulation of electron transport via said surfaces/interfaces. Discovered in 2006 and originally studied for strain sensing [15-17], significant progress has been made on piezotronics, both for deepening the research on strain sensing as well as for extending its applications to many relevant applications such as logic computation, chemical sensing, optoelectronics, tactile imaging and so on. This paper aims at reviewing the milestone progress in the field of piezotronics and also acts as a follow-up of the $2^{\text {nd }}$ International Conference on 
Nanogenerators and Piezotronics.

2. Fundamentals of piezotronics

The piezotronic effect is an effect that regulates charge carrier transport via the modulation of the band structure at contact interfaces. The piezopotential induced by mechanical triggering serves as a 'gate voltage' and a piezotronic device can be considered as a strain-gated transistor. With piezoelectric semiconductors as the functioning material, the piezotronic effect may take place at either the metal-semiconductor interface or the semiconductor-semiconductor interface. Both situations have been investigated in details [18-21] and their fundamentals will be briefly reviewed in the following.

2.1 Piezotronic effect on metal-semiconductor (M-S) contact interface

When a metal material and a semiconductor material come into contact, the Fermi level of the semiconductor will be aligned with that of the metal material, leading to redistribution of charges at the junction area. Depending on the types of materials, either Ohmic contact (non-rectifying junction) or Schottky contact (rectifying junction) may be formed. The piezotronic effect modulates the interfacial energy at the junction area. The modulation can be directly reflected by the effective resistance of the junction. Accordingly, the strength of this effect is positively related to the ratio of the junction resistance to the bulk resistance. In this regard, the existence of a Schottky barrier is the prerequisite for the piezotronic effect to take place at an M-S interface. By applying strain to the piezoelectric semiconductor, either positive or negative polarization charges are induced at the contact interface. Taking n-type semiconductor as an example, positive polarization charges attract free electrons and decrease the level of depletion, leading to reduced Schottky barrier height as well as its effective resistance; negative charges repel electrons and increase the level of depletion, leading to increased Schottky barrier height as well as its effective resistance. This process is schematically explained in Figure 1, which serves as the qualitative explanation of how the piezotronic effect modulates the electron transport across the M-S contact interface.

According to the diffusion theory, the current flowing through the Schottky barrier can be expressed as [22]

$J_{n} \approx J_{D} \cdot\left[\exp \left(\frac{q V}{\mathrm{kT}}\right)-1\right]$,

where the saturation current density $J_{D}$ can be expressed as

$J_{D} \approx q^{2} D_{n} N_{C} \cdot(k T)^{-1} \cdot \sqrt{\left[2 q N_{D}\left(\Psi_{b i}-V\right) \cdot \varepsilon_{s}^{-1}\right]} \cdot \exp \left[\frac{-\mathrm{q} \Phi_{B n}}{k T}\right]$.

In this equation, $D_{n}$ is the electron diffusion coefficient, $N_{C}$ is the effective density of states in the conduction band, $N_{D}$ is the donor concentration in the semiconductor, $\Psi_{b i}$ is the built-in potential, $\varepsilon_{s}$ is the permittivity of the semiconductor and $\Phi_{B n}$ is the Schottky barrier height. As explained earlier, the piezotronic effect is the tuning of the Schottky barrier height via piezoelectric charges. Thus this factor is further derived as: 
$\Phi_{\mathrm{Bn}}=\Phi_{\mathrm{Bn} 0}-q^{2} \rho_{\text {piezo }} W_{\text {piezo }}^{2}\left(2 \varepsilon_{s}\right)^{-1}$,

where $\rho_{\text {piezo }}$ is the density of polarization charges and $W_{\text {piezo }}$ represents the effective width of the polarization charge distribution. The correlation between the applied strain and the Schottky barrier height could then be established by

$P_{z}=e_{33} S_{33}=q \rho_{\text {piezo }} W_{\text {piezo }}$,

where $e_{33}$ is the piezoelectric coefficient and $s_{33}$ is the applied strain along the polar axis of the piezoelectric material. Ultimately, the relation between current density across the junction area and the applied strain is expressed as

$\mathrm{J}_{n} \approx J_{D 0} \cdot \exp \left[q e_{33} S_{33} W_{\text {piezo }} \cdot\left(2 \varepsilon_{s} k T\right)^{-1}\right] \cdot \exp \left[q V \cdot(k T)^{-1}-1\right]$, where $J_{D O}$ is the saturation current density without introducing the piezoelectric polarization.

The above equation is the quantitative explanation on how the applied strain in a piezoelectric semiconductor could modulate the Schottky barrier height at the M-S interface and thus regulate the electron transport.

2.2 Piezotronic effect on semiconductor-semiconductor (S-S) contact interface

When two pieces of semiconductor materials are brought into contact, their difference in Fermi levels will give rise to energy barriers at the contact interface. For the same reason, if polarization charges are introduced, the energy barrier height will be modulated accordingly. The piezotronic effect can take place at n-n junctions, $p-p$ junctions as well as p-n junctions. Here we use the model of a homogeneous $p-n$ junction as an example and only consider the piezoelectricity from the n-type material to explain the process.

The most important character of a $p$ - $n$ junction is a wide depletion region due to interdiffusion of holes and electrons. The strong built-in field not only enables significant optoelectronic applications such as light emitting diodes (LEDs) and solar cells, but also greatly reduces the screening effect on the strength of the piezoelectric field due to the lack of free charge carriers within the space charge region [23, 24]. If positive piezoelectric polarization charges are induced at the interface by the n-type semiconductor, some electrons transporting across the junction will be attracted, leading to a downward bending of the band edge profiles at the junction area; if negative piezoelectric polarization charges are induced at the interface by the n-type semiconductor, electrons transporting across the junction will be repelled, leading to an upward bending of the band edge profiles at the junction area. This process is schematically explained in Figure 2.

By using the abrupt junction model, the built-in potential could be expressed in a simple form as below [25]:

$\psi_{\mathrm{bi}}=q \cdot\left(2 \varepsilon_{s}\right)^{-1} \cdot\left(N_{A}(x) W_{D p}^{2}+\rho_{\text {piezo }} W_{\text {piezo }}^{2}+N_{D}(x) W_{D n}^{2}\right)$.

In this equation, $N_{A}(x)$ is the acceptor concentration, $N_{D}(x)$ is the donor concentration, 
$\rho_{\text {piezo }}$ is the density of polarization charges, $W_{D p}$ and $W_{D n}$ are the widths of depletion layers for the p-type side and n-type side respectively and $W_{\text {piezo }}$ is the equivalent distribution width of polarization charges. The built-in potential on one hand is directly related to the piezopotential generated by the piezoelectric semiconductor and on the other hand is the key parameter in optoelectronic applications. This clearly indicates how the piezotronic effect exerts its influence on optoelectronic processes.

Since M-S Schottky junction can be considered as a special p-n junction, we further assume that $p_{n 0} \gg n_{p 0}$, where $p_{n 0}$ is the thermal equilibrium hole concentration in the n-type semiconductor and $n_{p 0}$ is the thermal equilibrium electron concentration in the p-type semiconductor. Consequently we could apply the same expression that is used for the M-S contact:

$\mathrm{J}_{n} \approx J_{C 0} \cdot \exp \left[q e_{33} s_{33} W_{\text {piezo }} \cdot\left(2 \varepsilon_{s} k T\right)^{-1}\right] \cdot \exp \left[q V \cdot(k T)^{-1}-1\right]$, where $J_{C O}$ represents the saturation current density without introducing the piezoelectric polarization.

Taking LED as an example [26],

$P_{\text {optic }}=\beta\left\{J_{c 0} \cdot \exp \left[q e_{33} S_{33} W_{\text {piezo }} \cdot\left(2 \varepsilon_{s} k T\right)^{-1}\right] \cdot \exp \left[q V \cdot(k T)^{-1}-1\right]\right\}^{b}$

where $P_{\text {optic }}$ is the optical power output of the LED, $\beta$ is a constant decided by materials and device structures and $b$ is the power-law parameter. The external quantum efficiency (EQE) is $\eta_{e x}=\alpha \eta_{e x 0}$, where $\eta_{e x 0}$ is the EQE without piezopotential. The term $\alpha$ represents the piezo effect which can be expressed as

$\alpha=\left\{\exp \left[q^{2} \rho_{\text {piezo }} W_{\text {piezo }}^{2}\left(2 \varepsilon_{s} k T\right)^{-1}\right]\right\}^{b-1}$,

which reflects the modulation from the piezopotential on the carrier transport and the photo emission process.

The above analysis of the piezotronic effect on $\mathrm{p}-\mathrm{n}$ junctions gives the qualitative and quantitative explanation of the modulation process and the logic could be extended to more complicated scenarios that involve heterogeneous junctions, p-type piezoelectric semiconductors, intrinsic regions etc.

2.3 Influence of free carrier screening on the piezotronic effect

Of all the factors that influence the strength of the piezotronic effect, there are two that are mostly important, namely the piezoelectric coefficient, which dictates the original level of piezoelectric polarization, and the free carrier screening effect [27-30], which decides the effective level of piezoelectric polarization. While the first factor is straight forward, the second one can be very flexible which provides space to greatly improve the performance of piezotronic transistors. The free carrier concentration in semiconductors is decided by the type of dopants, the doping concentration and the excitation energy. Two major sources of excitation are the thermal energy and light energy. The effect of light on the piezotronic effect will be discussed later and in this section, works studying the dependence of the piezotronic 
effect on doping concentration and environmental temperature will be reviewed [31].

For a non-degenerate semiconductor with low levels of doping, its carrier concentration can be given by $\mathrm{n}=N_{c} \exp \left(-\frac{E_{C}-E_{F}}{k T}\right)$ for $\mathrm{n}$-type doping and $\mathrm{p}=$ $N_{V} \exp \left(-\frac{E_{F}-E_{V}}{k T}\right)$ for p-type doing, where $N_{C} \propto T^{3 / 2}$ is the effective density of states for the conduction band and $N_{V} \propto T^{3 / 2}$ is the effective density of states for the valence band [32]. Clearly the doping concentration which determines the value of ( $\left.E_{C}-E_{F}\right)$ or $\left(E_{F}-E_{V}\right)$ and the temperature are the two controlling parameters for the carrier concentration. On the other hand, for an n-type piezoelectric semiconductor, its positive polarization charges will be partially screened by the free electrons and for a p-type piezoelectric semiconductor, its negative polarization charges will be partially screened by the free electron holes. Therefore, we expect that lower doping concentration and lower temperature should lead to a stronger piezotronic effect.

Systematic experiments have been performed to verify the above theory [31]. $\mathrm{ZnO}$ nanowires were synthesized via physical vapor deposition and the products were divided into three groups: low, moderate and high doping concentration. Silver was used as the electrode material and aluminum foil coated with Kapton film was used as the substrate to provide insulation as well as decent thermal conductivity. Measurements were conducted in a micro manipulation cryogenic probe system which could provide an environmental temperature from $77 \mathrm{~K}$ to $300 \mathrm{~K}$. Firstly, the piezotronic effect of the device from the low doping concentration group was tested under different temperatures. Piezotronic response characteristics were obtained and the ones under $300 \mathrm{~K}, 200 \mathrm{~K}$ and $100 \mathrm{~K}$ are shown in Figure 3(a), (b) and (c) respectively. By comparison, we could see that the piezotronic effect is obvious under $300 \mathrm{~K}$ but become even stronger with decreasing temperature. Secondly, the piezotronic effect of the devices from three groups were tested and compared under the temperature of $77 \mathrm{~K}$, as shown in Figure 4 (a), (b) and (c) respectively. By comparison, we could see that the piezotronic effect became weaker with increasing doping concentration and even under a temperature as low as $77 \mathrm{~K}$, the carrier concentration in the high doping group still leads to heavy screening of the piezopotential. The experimental results exhibited here further verify the interpretation of the fundamental mechanism of the piezotronic effect and provide direct insights on how the screening effect impairs the piezotronic effect.

\section{Variations of single piezotronic transistors}

The study of a single piezotronic transistor is the key link between the theories of piezotronics and sophisticated applications. By experimenting with different types/forms of materials and different device configurations, we are not only able to further verify the theories, but also improve existing properties as well as introduce new properties to lay the foundations for novel applications. In this section, we will briefly review the variations of basic piezotronic transistors from the materials point 
of view and discuss the uniqueness and potentials of these configurations.

\subsection{Choice of channel materials}

The basic requirements of the channel material for piezotronic transistors, as mentioned previously, are being piezoelectric and semiconducting. There are additional factors to consider in practical applications for different purposes. Material synthesis is among the major concerns. Up to now, the majority of reports on the piezotronic effect use $\mathrm{ZnO}$ as the channel material and the number one reason behind that is the diversity and convenience in its synthesis. A lot of methods may be used to synthesize $\mathrm{ZnO}$ thin films and nanostructures such as chemical vapor deposition [33-35], metalorganic vapor phase epitaxy [36, 37], electrophoretic deposition [38, 39], pulsed laser deposition [40, 41], sputtering [42, 43], sol-gel synthesis [44, 45] and hydrothermal synthesis [46-49]. As with many semiconductor materials, the chemical vapor deposition is a low cost method that produces high quality nanowires. With a single zone high temperature tube furnace, $\mathrm{ZnO}$ nanowires array or ultra-long single nanowires could be easily synthesized and engineered by using different sources and substrates [33-35]. More importantly, $\mathrm{ZnO}$ nanostructures and thin films can be synthesized hydrothermally under $100{ }^{\circ} \mathrm{C}$ with simple chemical reactions, leading to great controllability and flexibility [46-49]. This convenience of synthesizing $\mathrm{ZnO}$ nanostructures is becoming more and more important under the context of rapidly developing flexible electronics, where the compatibility of the synthesis method and polymeric substrates is extremely important. Other typical piezoelectric semiconductors such as $\mathrm{CdS}, \mathrm{CdSe}, \mathrm{InP}$ and $\mathrm{GaN}$, are more or less restricted by their lack of low temperature synthesis measures and economic, effective vapor phase synthesis methods $[50,51]$.

In addition to synthesis issues, the materials' electrical, optical and chemical properties are also critical criteria to consider for the selection of channel materials. As mentioned above, the doping concentration is the major factor that influences the performance of piezotronic transistors. Therefore, it is desired that the materials' doping could be easily manipulated. The doping of $\mathrm{ZnO}$ is strongly correlated with oxygen partial pressure in the environment and could thus be manipulated by measures of annealing and plasma processing in addition to in-growth doping [41, 52, 53]. For non-oxide piezoelectric semiconductors, however, such manipulation relies on more sophisticated measures such as ion implantation. As for the optical properties, notable factors include the materials' band gaps and their corresponding absorption peaks. With $\mathrm{ZnO}, \mathrm{GaN}$ in the UV range, $\mathrm{CdS}$ in the visible range and CdTe in the IR range, the optical spectrum is well covered and we can select materials according to needs to build photo detectors, LEDs and solar cells and then use strain induced piezopotential to modulate junction interfaces for novel effects and functions [54, 55]. As for chemical properties, the level of chemical activeness and inertness is usually considered. Chemical activeness is desired if chemical sensing is to be coupled with the piezotronic effect and chemical inertness is desired if piezotronic devices are required to operate under harsh environments. For example, $\mathrm{ZnO}$ responses to both 
oxidizing and reducing gas $[47,56]$ and its gas sensing process could be coupled with the piezotronic effect for enhanced sensitivity. $\mathrm{GaN}$, on the other hand, is more chemically stable than $\mathrm{ZnO}$ and may be used under humid and corrosive environments [57].

\subsection{P-type vs n-type channel materials}

Currently the majority of works on piezotronics use $\mathrm{ZnO}$ as the channel material. However, p-type doping of $\mathrm{ZnO}$ has been difficult $[58,59]$. As a result, few works study the piezotronic effect based on p-type channel materials. However, doing research in this aspect is very important since it not only improves our understanding of the piezotronic effect on electron hole transport, but also enables piezotronics on homogenous p-n junctions that would be important in both electronics and optoelectronics.

In theory, p-type channel piezotronic transistors should work in a similar manner as that of n-type channel piezotronic transistors. Relevant band diagrams for the former are shown in Figure 5. There are several distinctions for the case of p-type semiconductors from n-type ones: the bend structure bends downward when the semiconductor has a higher work function than the metal, leading to the formation of the Schottky barrier; positive polarization charges increase the barrier height and negative polarization charges decrease the barrier height; negative polarization charges get partially screened by the free electron holes while positive polarization charges are not affected. These characters, being opposite to that of the n-type case, do not lead to any essential differences in the working principle.

The first p-type piezotronic transistor was reported in 2013 [60], roughly 7 years after the discovery of piezotronics. It was made possible by successfully synthesizing $\mathrm{Sb}$ doped p-type ultra-long $\mathrm{ZnO}$ nanowires via the hydrothermal method. The success of doping was confirmed by both the TEM characterization and the FET characterization. Stability of the doping was also demonstrated by measuring the I-V characteristics of the p-type $\mathrm{ZnO}$ based FET after 6 months of synthesis and the p-type character was maintained. Measurement results for the FET and the piezotronic characterization is shown in Figure 6 (a) and (b), providing the first direct evidence that p-type piezotronic transistors work in the way predicted by theories.

However, at the current stage, it is still preferred to use n-type $\mathrm{ZnO}$ to build piezotronic devices when the conduction type is irrelevant. The reason is that the stability of p-type doping of $\mathrm{ZnO}$ nanowires is still unclear on the scale of years [61, 62] and the p-type doping of $\mathrm{ZnO}$ thin films is even more difficult due to smaller aspect ratios. Using dopants with large atomic radius such as $\mathrm{Sb}$ is also problematic. They distort the crystal structure of the $\mathrm{ZnO}$, leading to undesired situations such as reduced growth length and degraded material flexibility [60, 63]. GaN, on the other hand, has no problem of being doped into both n-type and p-type and has proven advantages in the field of optoelectronics $[64,65]$. Therefore, research on developing 
p-type GaN piezotronic devices based on both M-S junctions and homogeneous p-n junctions should be considered.

3.3 Nanowire vs thin film vs 2-D channel materials

The field of piezotronics was initiated by using $\mathrm{ZnO}$ nanowires and in a long period of time after that, the choice of materials were still limited to 1-D nanomaterials. One of the considerations behind this phenomenon is that as-synthesized nanowires are usually highly crystalline [35, 66], which ensures that the piezoelectric field vectors within each unit cell are perfectly aligned, which leads to strong piezoelectricity on the macro-scale. In addition, 1-D nanomaterials have excellent mechanical properties and can endure a high level of straining before failure [67, 68]. However, 1-D nanomaterials do have their limitations. The lack of uniformity in synthesis methods is an evident problem [69, 70]. Discrepancies in material dimensions, morphologies and doping levels mean that devices made from single nanowires will have large performance variations, which strongly discourage the industry to adopt them due to very low yield. Additionally, it is also difficult to effectively assembly nanowires into orderly manners for further circuit integration [71, 72]. Under this context, piezotronic devices based on thin films were developed [73, 74]. The first practical piezotronic thin film device was developed based on RF sputtered ZnO. Since RF sputtering produces amorphous or polycrystalline thin films, normally speaking, the random orientation of crystalline grains will eliminate any macro-scale piezoelectricity. $\mathrm{ZnO}$, however, is a special material that has the property of self-texturing even during unheated sputtering process $[75,76]$. This leads to oriented growth, during which the c-axis of most grains are aligned along the growth direction. This feature instantly removes two major obstacles in implementing the piezotronic effect with thin films: the difficulty in synthesizing monocrystalline thin films and its incompatibility with flexible electronics. The level of crystallinity, grain alignment and surface conductance could be further adjusted by sputtering parameters, annealing and plasma treatment and an effective thin film piezotronic device could be produced after optimizations [74, 77]. TEM images of the $\mathrm{ZnO}$ thin film and the device's piezotronic characteristics are shown respectively in Figure 7 (a) and (b). The progress in realizing thin film based piezotronic transistors not only expands the selection of materials in this field, but also makes it easier for the industry to accept this relatively new concept and incorporate this field into development of future consumer electronics.

The third form of material that has been intensively studied in recent years is the atomically thick 2-D materials $[78,79]$. They have also come into sight in the field of piezotronics. These materials bring many extraordinary properties unheard of in traditional materials. In terms of piezoelectricity, traditional crystallography rules that of the thirty two crystal classes, twenty exhibit piezoelectricity due to the lack of symmetry center. This is no longer the case for 2-D materials since some asymmetries concealed in bulk materials are revealed for one layer or few layer structures $[80,81]$. One notable example is 2-D $\mathrm{MoS}_{2}$, which has been a star material recently due to the 
existence of a bandgap, unavailable in graphene. It thus has great potentials in the production of ultra-high performance electronic transistors and photodetectors. By further incorporating the piezotronic effect due to its piezoelectricity, we expect another research boom coming for 2-D materials that may bring substantial progress in piezotronics and related technologies.

4 Novel applications of piezotronic devices beyond strain sensors

The piezotronic effect is a great discovery not only because it provides a third strain sensing mechanism in addition to piezoresistance and piezoelectricity, but also because its potentials reach far beyond the field of strain sensing. In this section, examples of works of utilizing this effect for creative applications will be reviewed $[53,82]$.

\subsection{Piezotronic logic devices}

We have been using the word transistor for piezotronic devices from the beginning since we consider the strain induced piezopotential as a special type of gate voltage. Therefore, efforts are made to move a step forward to use these strain-gated transistors to build basic logic computation units. Noted that unlike traditional logic computation, the input here is the strain and the output is voltage. So a logic definition of the input is needed. As shown in Figure 8, the piezotronic transistor has increased conductance under tensile strain and has decreased conductance under compressive strain. Thus the tensile strain is assigned as logic input " 1 " and the compressive strain is assigned as logic input " 0 " so that the input is consistent with the output. A strain logic converter is then built by holding two piezotronic transistors back to back. Applying tensile strain to one device meant applying compressive strain for the other and logic input " 0 " and " 1 " should be defined according to one device. By connecting the circuit as shown in Figure 9 (a), the strain-voltage transfer diagram and the logic table was obtained, as shown in Figure 9 (b), which meant that when tensile strain (logic input "1") was applied, the whole device was turned off and when compressive strain (logic input "2") was applied, the whole device was turned on. This behavior is opposite to that of a single piezotronic transistor, meaning that a strain-gated logic inverter is achieved. By using other types of configuration, basic logic gates such as NAND, NOR and XOR could all be realized. This work shows the prospective of integrating this type of strain-electronics logic computation into human-machine interfacing technologies to spur novel applications.

\subsection{Piezotronic programmable resistive memories}

Resistive memory is a type of non-volatile memory with very fast switching speed and very small device size. Its structure is as simple as a dielectric material sandwiched between two electrodes. The device can be switched on and off by setting and resetting filament conduction paths $[83,84]$. $\mathrm{ZnO}$, along with other metal oxides such as $\mathrm{TiO}_{2}$ and $\mathrm{HfO}_{2}$ with wide band gaps, are the typical materials employed to build such memories [85, 86]. The addition of piezoelectricity in $\mathrm{ZnO}$ inspires interests in seeing how piezotronics would influence the resistive switching process. 
A single $\mathrm{ZnO}$ nanowire with its two ends placed on gold electrode pads supported by a flexible PET substrate was used as the study object. Due to the geometry of the nanowire, it was unlikely to form a thorough filament path across the material. Instead, partial filaments may be formed that would change the resistance of M-S contacts [87, 88]. In Figure 10 (a), the green curve corresponds to the case where the device was unstrained. It was shown that the device exhibited a bipolar switching behavior with its threshold voltage at around $+5 \mathrm{~V}$ and $-5 \mathrm{~V}$ respectively. By applying a tensile strain of $1.17 \%$, opposite polarization charges were induced on opposite polar surfaces of the nanowire, rendering a shift of the switching characteristics by the amount of -1.49 V. On the contrary, compressive strain of $0.76 \%$ was applied, rendering a shift of the switching characteristics by the amount of $1.18 \mathrm{~V}$. In Figure 10 (b), it shows the relationship between the applied strain and the shifts of threshold voltages on both sides and it can be concluded that the shifting of the two sides is almost identical and is linear to the applied strain. On the other hand, the ratio of high resistance to low resistance remains unaffected by the shifting.

The interesting coupling between the piezotronic process and the resistive switching process offers a mechanism of programming the threshold voltages of a memory device via mechanical perturbation. The device could not only be switched by applying varying voltage, but could also be switched under a fixed voltage but under different strains, adding a novel way of memory operation.

\section{Coupling of the piezotronic effect with other interfacial effects}

Coupling is a very important concept in physics and a very common phenomenon in nature. If a physician's job is to decouple complex phenomena into separate, explicit physical laws, then an engineer's job is to wisely couple selected physical laws to produce devices and systems of improved and new functions. As we know, the piezotronic effect is an interfacial effect that can both increase and decrease the interfacial energy barrier. It is thus of great interest to study the coupling of the piezotronic effect with other interfacial effects so that its applications could be diversified. There are many interfacial effects, including the resistive switching phenomenon that was just mentioned. In this section, we will review studies on the coupling of piezotronics with two other extremely important effects that involve interfacial process: the optoelectronics and the chemical sensing.

\subsection{Piezophototronics}

Optoelectronics is the study of using electronic devices to produce, harvest and control light. Electroluminescence and photovoltaics are the two major effects adopted in optoelectronic devices. Being reverse processes to each other, both utilize the p-n junction or its derivatives to transfer energy between electrons, holes and photons. By further introducing piezoelectricity into the region, we have a three way coupling effect that is named as the piezophototronic effect [14, 21, 27]. Light emitting diodes (LED) and solar cells are currently the two most important types of 
optoelectronic devices and in this section, we will review on how piezophototronics could improve their performances [77, 89].

5.1.1 Enhanced efficiency in inorganic/organic hybrid LEDs by piezophototronic effect

The efficiency of a LED depends on the carrier injection, recombination and the light extraction. Maintaining a balanced injection is the key to high emission efficiency. However, for heterogeneous junctions, due to different material properties and band alignment, it is usually difficult to inject equal amount of electrons and holes into the space charge region. The piezophototronic effect, however, can be used to tune this process.

Here, n-type $\mathrm{ZnO}$ and p-type PEDOT:PSS were used to construct a hybrid LED as show in in Figure 11 (a). Half of the $\mathrm{ZnO}$ NW was uniformly coated by the PEDOT:PSS and was placed onto separate gold electrode pads supported by a Kapton substrate. Strain was applied by bending the substrate and simulated results of the piezopotential distribution shown in Figure 11 (b) and (c) confirms that positive and negative piezo-charges could be induced respectively at the interface of the hetero-junction. By applying strain from tensile $0.003 \%$ to compressive $-0.008 \%$, the forward biased current continuously decreased as shown in Figure 11 (d) and the reversely biased current continuously increased as shown in Figure 11 (e). The asymmetry in this trend of changing indicates that the modulation is a result of the piezophototronic effect. The relationship between the forward injection current and the applied strain is shown in Figure 11 (f). In the meanwhile, the emission intensity and the corresponding efficiency was measured and calculated and the result is shown in Figure $11(\mathrm{~g})$. From tensile strain of $0.003 \%$ to compressive strain of $-0.008 \%$, although the injection current decreases, the emission intensity increases and consequently the external quantum efficiency is increased to as much as $230 \%$ by simply manipulating the strain applied on the $\mathrm{ZnO}$ nanowire. Visualization of the enhancement process was also provided by a CCD camera as shown in Figure 11 (h).

The mechanism behind this could be explained by the theories reviewed under "Piezotronic effect on semiconductor-semiconductor (S-S) contact interface". It has been explained that the piezo-polarization charges at the interface may create dips or bumps on the junction band structure. For the case of the hybrid LED presented here, more electrons were injected than holes, which led to the large current and low emission. By trapping the holes with the dip in the band structure and reducing electron injection with the reversely biased Schottky junction between $\mathrm{ZnO}$ and $\mathrm{Au}$ electrode, the injection was tuned toward balance and a larger efficiency as well as a stronger emission was achieved even with a smaller injection current.

\subsubsection{Enhanced efficiency in $\mathrm{ZnO} / \mathrm{P} 3 \mathrm{HT}$ solar cells by piezophototronic effect}

The efficiency of solar cells is determined by many factors such as reflection loss, semiconductor band width, carrier separation and carrier collection. The 
piezophototronic effect will assist in the carrier separation process by creating energy 'bumps' in the p-n junction area. A number of solar cell systems as well as junction based photo detection systems have been studied to confirm this mechanism. The n-type $\mathrm{ZnO}$ and p-type P3HT is a viable combination for solar cells due to the appropriate band gap of P3HT and its band alignment with $\mathrm{ZnO}$. The solar cell with the structure shown in Figure 12 (a) was adopted for the study. ITO was used as the electrode for $\mathrm{ZnO}$ film and $\mathrm{Au}$ was used as the electrode for P3HT film, both forming Ohmic contact with respective semiconductors. Sun light went through the visibly transparent ITO and $\mathrm{ZnO}$ layers and reach the p-n junction to excite electron-hole pairs. A series of strains was applied onto the flexible substrate .As shown in Figure 12 (b), the short circuit current could be increased by tensile strain and decreased by compressive strain while the open circuit voltage remains unaffected. According to $V_{O C} \approx \frac{\mathrm{nkT}}{q} \operatorname{Ln}\left(\frac{J s c}{J o}\right), 1 \mathrm{~mA} / \mathrm{cm}^{2}$ change in short circuit current density leads to approximated $1 \times 10^{-4} \mathrm{~V}$ change in open circuit voltage. This means that the theory is in consistent with the tuning observed here. By improving the crystallinity and adjusting the doping concentration of the $\mathrm{ZnO}$ film, a much stronger tuning effect could be observed after optimization, as shown in Figure 12 (c). Compared with the case in Figure 12 (b) that the efficiency was increased by $3.456 \%$ under $0.32 \%$ tensile strain, the efficiency corresponding to Figure 12 (c) was increased by $12.1 \%$ under $0.32 \%$ tensile strain. The reason for the stronger piezophototronic effect is a more balanced piezoelectricity between polarization charge density and thin film conductivity. The principle of enhancement and the guideline in optimizing film properties presented in this work could be further applied to other types of thin film solar cells involving piezoelectric semiconductors such as CdTe, GaAs etc.

\subsection{Enhanced chemical sensing by piezotronics}

Chemical sensing by semiconductor devices is usually achieved by the adsorption of chemical species onto the semiconductor material and their subsequent interaction with the free charge carriers $[90,91]$. For example, if certain chemicals are adhered to the semiconductor surface and become negatively charged, they in effect apply a negative voltage to the semiconductor. The voltage that is applied onto the bulk part of the semiconductor affects the conduction channel width, thus regulating the carrier transport in a similar way as that of a FET. The voltage that is applied onto the junction area of the semiconductor device affects the energy barrier height, thus regulating the carrier transport in a similar way as that of a piezotronic transistor. Therefore, for a chemical sensor using piezoelectric semiconductor materials and with Schottky junctions at the M-S interface, we could introduce the piezotronic effect to enhance the sensitivity.

$\mathrm{ZnO}$ is a typical material for oxygen sensing due to its abundant oxygen vacancies. However, the sensing mechanism could only be activated at temperatures higher than $150{ }^{\circ} \mathrm{C}[91,92]$. Assisted by the piezotronic effect, room temperature sensing was achieved, making the $\mathrm{ZnO}$ oxygen sensor much more practical. A vacuum system 
with a mechanical feed through was used to conduct the test so that strain could be applied to the device while maintaining a stable gas environment isolated from the atmosphere. Various levels of oxygen was introduced into the chamber. At each oxygen partial pressure level, a full set of piezotronic characterization was performed. To reflect accurate sensitivity, the oxygen sensor was reset after each group of measurements by UV illumination. Data for the sensor under the bias voltage of $1 \mathrm{~V}$ is compiled in Figure 13 (a) and (b). It could be seen that while the absolute current decreases with increasing tensile strain, the relative current change, namely the sensitivity, increases with increasing tensile strain at a fixed oxygen partial pressure. Similarly, the data for the sensor under the bias voltage of $-1 \mathrm{~V}$ is also complied, as shown in Figure 13 (c) and (d) and similar trends can be observed. This means that by inducing piezo-polarization charges at the M-S junction interface, we could optimize the energy barrier height so that the device could be more responsive to the adsorption of oxygen species. The device had a back to back Schottky diode structure and the phenomenon that the enhancement for the device under positive bias was more obvious than that under negative bias indicated that the two Schottky diodes were asymmetric and had different initial barrier heights.

The general principle of piezotronic enhancement of chemical sensors could be applied for other chemical sensing not only for gas species but also for chemicals in liquid environment, as demonstrated by other works [93, 94].

6 Integration of piezotronic devices towards functional systems

The development of nanotechnology goes through three stages: the synthesis and obtaining of nanomaterials: the demonstration on improved or novel properties of the nanomaterials; the organizing and integration of individual devices into functional systems. Nanotechnology research has gradually entered into the third stage on many fronts. The obstacle and difficulty that is faced is the incompatibility between the existing microfabrication technologies and the processing of nanomaterials, via the bottom-up approach instead of the top-down approach. Special strategies have been developed to tackle this problem. In this section, we will review breakthroughs specifically for the integration of piezotronic units that enables important applications in tactile imaging and electronic interfacing $[95,96]$.

6.1 3D piezotronic transistors matrix for electrical imaging of pressure distribution Piezotronics as a novel strain/force/pressure sensing mechanism has been demonstrated by many works using a single transistor or a few transistors. However, the potential of this new effect may not be fully revealed without the demonstration of pressure mapping by arrayed transistors. On the other hand, pressure imaging has significant applications in human-electronic interfacing. Specifically, high resolution and high sensitivity pressure imagers are greatly needed to mimic the sense of touch to be used for prosthesis, robotics and medications, which are difficult to achieve with piezoresistive and piezoelectric strain sensors. 
To cater the above need, strain-gated vertical piezotronic transistors array have been developed. The device structure is shown in Figure 14 (a). Crossbar electrodes are used to simplify electrical interconnects while maintaining the capability to individually address each unit device. Sandwiched in between are arrays of vertically aligned $\mathrm{ZnO} \mathrm{NWs}$ as the active channels for piezotronic transistors. The strain-gated transistor does not need an actual third gate and this advantage greatly simplifies the integration process of the matrix. Equivalent circuit of the device is shown in Figure 14 (b) and a photo image of a real device is shown in Figure 14 (c), demonstrating its transparency.

The actual device was designed to have 92 rows and 92 columns of electrodes and thus have 8464 transistors in total on an area of $1 \mathrm{~cm}^{2}$. The density of pressure sensors already exceeds that of human finger tips at $240 \mathrm{~cm}^{-2}$ [97]. Fabrication of the array device was achieved by combining the conventional microfabrication techniques with the hydrothermal synthesis of aligned NWs array. UV lithography was used to define all the patterns and electron beam evaporation was used for metallization. The ordered synthesis was achieved by tightly controlling the growth parameters as well as predefining the nucleation locations. SU-8 was used to separate the bottom and top electrodes and also encapsulating the $\mathrm{ZnO}$ NWs. Selective pictures of the device structure during different fabrication stages are shown in Figure 15.

Electrical characterization of the device matrix was done by using two multi-channel high speed switches, which enable the sequential addressing of all the pixel transistors in time. Line scan of one row of pixels was took out to study the piezotronic characteristics, as shown in Figure 16 (a). Here, the first row of figures are the topological images of 23 selected pixels. The second and the third rows of figures are the current values of these pixels under a bias voltage of $1 \mathrm{~V}$ without and with externally applied strain respectively. The two pixels that have reduced current as a result of the applied strain is marked with blue background. The second and third row of data was recompiled in Figure 16 (b) to better reflect the current change over time. It could be seen that the time segments highlighted in blue are the ones with applied strain which shows the dynamic response of the pixels. For one specific pixel numbered 46, its response to various levels of strain was further analyzed, as shown in Figure 16 (c). The pixel device could effectively differentiate a few kPa's pressure difference from $0 \mathrm{kPa}$ to $\sim 30 \mathrm{kPa}$ but then reaches saturation. Its sensitivity and sensing range is reflected in the upper right inset which shows that the current under fixed bias decreases with increasing pressure. The sensitivity of the device is calculated to be $2.1 \mu \mathrm{SkPa}^{-1}$.

The pressure imaging capability of the device matrix is subsequently characterized. Firstly, the distribution of the initial conductance of all the transistors is obtained, shown in Figure 17 (a). 95\% of the transistors have their current fall in the range of $13.7 \pm 2.73 \mu \mathrm{A}$, demonstrating decent uniformity. A hexagonal object was then pressed against the device and the whole device was scanned to obtain a new current 
matrix. By subtracting the initial one from the new one, the current metrology map in Figure 17 (b) is obtained, clearly reflecting the distribution of applied strain by the relative current change at each pixel. Multi-dimensional pressure imaging was then demonstrated by using a small object to sequentially apply pressure on different locations of the matrix device. Firstly, the same pressure was applied onto six different locations to draw the shape of a letter ' $A$ ' and the corresponding result was shown in Figure 17 (c1). Then the process was repeated with the difference of applying a higher pressure on two locations and the corresponding result was shown in Figure 17 (c2). The results indicate that the device can not only tell the coordinate of the applied pressure but also register the value of the pressure so that pressure imaging can be achieved.

The above work not only demonstrated the integration of piezotronic transistors, but also revealed the strong potential of this technology being used for high resolution, high sensitivity pressure imaging that could lead to major breakthroughs in mimicking the sense of touch for very important applications such as prosthesis, robotics, medications etc. for human-electronic interfacing.

6.2 3D piezophototronic LEDs for electroluminescent imaging of pressure distribution As reviewed in previous sections, the piezophototronic effect can be used to modulate the emission intensity of piezoelectric semiconductor based LEDs. By considering from another angle, this effect could also be used for pressure sensing, where the output signal is the varying light emission as a result of varying input pressure. Compared with detecting pressure by measuring current change, this method does not require complex interconnects since the transport of light signal does not depend on any media. On the other hand, the processing of the light signal requires special optical processors that are still under development. Nevertheless, the study of using piezophototronic LEDs for pressure imaging certainly has significant meanings for expanding the field of piezotronics for future generations of interfacing technologies.

The structure of the device is shown in Figure 18 (a). The p-type semiconductor is the $\mathrm{GaN}$ thin film grown on a sapphire substrate. The n-type semiconductor is the $\mathrm{ZnO}$ nanowires array epitaxially grown on GaN. PMMA was applied to infiltrate the space among the NWs, acting both as a buffering material and an insulating material. Ni/Au and ITO electrodes were fabricated to form Ohmic contact with the GaN film and the $\mathrm{ZnO}$ NWs respectively. Particularly, both electrodes were continuous without patterning, which greatly reduced the complexity of the fabrication. The growth of the ordered NWs array, as shown in Figure 18 (b), was achieved by high resolution patterning of $\mathrm{ZnO}$ seed layers and the subsequent hydrothermal growth. The well-defined structures of the NWs indicate their single crystallinity. The center to center distance between two adjacent NWs is $4 \mu \mathrm{m}$, achieving a pixel resolution of $6350 \mathrm{dpi}$, which would be extremely difficult to achieve by using electronic methods of pressure sensing. 
Each $\mathrm{ZnO}$ nanowire acted as an individual light emitter and the LEDs array were lit up by applying a $5 \mathrm{~V}$ forward bias voltage, as shown in Figure 18 (c). The intensity of these LEDs were monitored by a CCD camera. Five examples of them were examined and their light intensity was shown in Figure 18 (d), indicating the uniformity of the emission. By applying pressure to the device, compressive strain was applied to the $\mathrm{ZnO} \mathrm{NWs}$ which were grown along their positive c-axis. Positive polarization charges were then induced at the GaN-ZnO p-n junction interface, leading to a dip in the band diagram as explained in previous sections. As a result, the recombination rate of the electrons and holes were elevated, leading to increased light emission intensity under the same bias voltage. The dynamic test of the LEDs was shown in Figure 18 (e), where the pressure was periodically applied. The current flowing through the LEDs was periodically increased as a result of the enhanced light emission. The response and the relaxation time of such a process was less than $0.1 \mathrm{~s}$, demonstrating the fast switching capability of the pressure sensors. The behavior of a single LED was monitored and as shown in Figure 18 (f), its emission intensity gradually increased as a result of increasing compressive strain. At the strain of $-0.15 \%$, the enhancement could reach $300 \%$.

A convex character pattern of "PIEZO" was used to apply pressure to study the pressure mapping ability of the device. Figure 19 (c) is a CCD picture of the LEDs array under $5 \mathrm{~V}$ forward bias without pressure. Then a pressure that corresponded to a compressive strain of $-0.06 \%$ was applied and the emission picture was shown in Figure 19 (d). By further increasing the strain level to $-0.15 \%$, the image of the lit up LEDs array was obtained in Figure 19 (e). It could be seen that the pattern of "PIEZO" became clearer with higher levels of strain and by subtracting the base emission intensity, a pressure image was successfully obtained, shown in Figure 19 (f). The signal to noise ratio was further analyzed by comparing the LEDs affected and unaffected by the strain. The behavior of the LEDs marked by a vertical pink dash line in Figure 19 (d) was monitored and their relative emission enhancement is shown in Figure $19(\mathrm{~g})$. The behavior of the LEDs marked by a horizontal red dash line in Figure 19 (d) was also monitored and their relative emission change is shown in Figure 19 (h). By comparison, the LEDs under pressure showed enhancement by a factor of 4-5 while the LEDs under no pressure only showed variations of less than $10 \%$ on average in their emission intensity.

The advantages of this novel pressure imaging device is its succinct structure, simple electrode fabrication, fast response and ultrahigh resolution. If integrated with future optical computations, this technology may enable microscale force and pressure mapping that would otherwise be extremely difficult to achieve.

\section{Summary and prospects}

In summary, the piezotronic effect regulates charge carrier transport across junction barriers by inducing piezo-polarization charges from piezoelectric semiconductors. The discovery of this effect changes the situation where the study of piezoelectricity 
was dominated by insulators. The physical level interaction between piezoelectricity and electronics has thus been studied for the first time. The unique advantage of this effect offers a great alternative to piezoelectricity and piezoresistivity to build strain/force/pressure sensors and sensing systems. More importantly, as an interfacial effect that manipulates the junction barriers, it can be coupled with many other interfacial effects which lead to new phenomena as well as novel applications. Mechanics, photonics, chemistry, electromechanics, optoelectronics, electrochemistry and even biology are all the fields that can be connected with piezotronics. Its scope is so broad that a new field of study is quickly taking shape in the research community. With the excellent work already done on piezotronics, there is a strong reason to believe that by going down this road persistently, this technology will eventually make major impacts and even lead to revolutions for applications of multi-functional sensing systems, human-electronics interfacing, MEMS, energy harvesting and so on.

Acknowledgements: Research was supported by BES DOE, NSF, Airforce, Samsung, SKKU (Korea), MANA NIMS (Japan), and the Knowledge Innovation Program of the Chinese Academy of Sciences (KJCX2-YW-M13), the Hightower Chair foundation, and the "thousands talents" program for pioneer researcher and his innovation team, China. We sincerely thank the following colleagues whose works significantly contributed to the field of piezotronics (not in particular order): Xudong Wang, Jun Zhou, Yan Zhang, Ying Liu, Simiao Niu, Ken Pradel, Ruomeng Yu and many others.

\section{References:}

[1] D.H. Kim, N.S. Lu, R. Ma, Y.S. Kim, R.H. Kim, S.D. Wang, J. Wu, S.M. Won, H. Tao, A. Islam, K.J. Yu, T.I. Kim, R. Chowdhury, M. Ying, L.Z. Xu, M. Li, H.J. Chung, H. Keum, M. McCormick, P. Liu, Y.W. Zhang, F.G. Omenetto, Y.G. Huang, T. Coleman, J.A. Rogers, Science, 333 (2011) 838-843.

[2] Y.G. Sun, J.A. Rogers, Adv Mater, 19 (2007) 1897-1916.

[3] J.H. Ahn, J.H. Je, J Phys D Appl Phys, 45 (2012).

[4] F. Carpi, D. De Rossi, leee T Inf Technol B, 9 (2005) 295-318.

[5] B.Z. Tian, J. Liu, T. Dvir, L.H. Jin, J.H. Tsui, Q. Qing, Z.G. Suo, R. Langer, D.S. Kohane, C.M. Lieber, Nat Mater, 11 (2012) 986-994.

[6] P.S. Waggoner, H.G. Craighead, Lab Chip, 7 (2007) 1238-1255.

[7] W.P. Eaton, J.H. Smith, Smart Mater Struct, 6 (1997) 530-539.

[8] A.A. Barlian, W.T. Park, J.R. Mallon, A.J. Rastegar, B.L. Pruitt, P leee, 97 (2009) 513-552.

[9] Z.L. Wang, J Phys-Condens Mat, 16 (2004) R829-R858.

[10] S.P. Beeby, M.J. Tudor, N.M. White, Meas Sci Technol, 17 (2006) R175-R195.

[11] A. Janshoff, H.J. Galla, C. Steinem, Angew Chem Int Edit, 39 (2000) 4004-4032.

[12] Z.L. Wang, Mat Sci Eng R, 64 (2009) 33-71.

[13] J. Zhou, Y.D. Gu, P. Fei, W.J. Mai, Y.F. Gao, R.S. Yang, G. Bao, Z.L. Wang, Nano Lett, 8 (2008) 3035-3040.

[14] Z.L. Wang, J Phys Chem Lett, 1 (2010) 1388-1393.

[15] X.D. Wang, J. Zhou, J.H. Song, J. Liu, N.S. Xu, Z.L. Wang, Nano Lett, 6 (2006) 2768-2772.

[16] J.H. He, C.L. Hsin, J. Liu, L.J. Chen, Z.L. Wang, Adv Mater, 19 (2007) 781-+. 
[17] Z.L. Wang, Adv Mater, 19 (2007) 889-892.

[18] Y. Zhang, Y. Liu, Z.L. Wang, Adv Mater, 23 (2011) 3004-3013.

[19] Z.Y. Gao, J. Zhou, Y.D. Gu, P. Fei, Y. Hao, G. Bao, Z.L. Wang, J Appl Phys, 105 (2009).

[20] Y. Gao, Z.L. Wang, Nano Lett, 9 (2009) 1103-1110.

[21] Y. Liu, Q. Yang, Y. Zhang, Z.Y. Yang, Z.L. Wang, Adv Mater, 24 (2012) 1410-1417.

[22] C.R. Crowell, S.M. Sze, Solid State Electron, 9 (1966) 1035-\&.

[23] J. Shi, M.B. Starr, X.D. Wang, Adv Mater, 24 (2012) 4683-4691.

[24] J. Shi, M.B. Starr, H. Xiang, Y. Hara, M.A. Anderson, J.H. Seo, Z.Q. Ma, X.D. Wang, Nano Lett, 11 (2011) 5587-5593.

[25] R.S. Muller, T.I. Kamins, M. Chan, Device electronics for integrated circuits, 3rd ed., John Wiley \& Sons, New York, NY, 2003.

[26] Y. Zhang, Z.L. Wang, Adv Mater, 24 (2012) 4712-4718.

[27] Z.L. Wang, Nano Today, 5 (2010) 540-552.

[28] T. Mukai, M. Yamada, S. Nakamura, Jpn J Appl Phys 1, 38 (1999) 3976-3981.

[29] H. Gotoh, T. Tawara, Y. Kobayashi, N. Kobayashi, T. Saitoh, Appl Phys Lett, 83 (2003) 4791-4793.

[30] T. Kuroda, A. Tackeuchi, J Appl Phys, 92 (2002) 3071-3074.

[31] Y.F. Hu, B.D.B. Klein, Y.J. Su, S.M. Niu, Y. Liu, Z.L. Wang, Nano Lett, 13 (2013) 5026-5032.

[32] R. Enderlein, N.J.M. Horing, World Scientific, Singapore ; River Edge, N.J., 1997, pp. 1 online resource (xvi, 771 pages).

[33] M.H. Huang, S. Mao, H. Feick, H.Q. Yan, Y.Y. Wu, H. Kind, E. Weber, R. Russo, P.D. Yang, Science, 292 (2001) 1897-1899.

[34] X.D. Wang, J.H. Song, P. Li, J.H. Ryou, R.D. Dupuis, C.J. Summers, Z.L. Wang, J Am Chem Soc, 127 (2005) 7920-7923.

[35] J.H. Song, X.D. Wang, E. Riedo, Z.L. Wang, Abstr Pap Am Chem S, 231 (2006).

[36] Y. Liu, C.R. Gorla, S. Liang, N. Emanetoglu, Y. Lu, H. Shen, M. Wraback, J Electron Mater, 29 (2000) 69-74.

[37] M. Willander, O. Nur, Q.X. Zhao, L.L. Yang, M. Lorenz, B.Q. Cao, J.Z. Perez, C. Czekalla, G. Zimmermann, M. Grundmann, A. Bakin, A. Behrends, M. Al-Suleiman, A. El-Shaer, A.C. Mofor, B. Postels, A. Waag, N. Boukos, A. Travlos, H.S. Kwack, J. Guinard, D.L. Dang, Nanotechnology, 20 (2009).

[38] E.M. Wong, P.C. Searson, Appl Phys Lett, 74 (1999) 2939-2941.

[39] C.S. Rout, S.H. Krishna, S.R.C. Vivekchand, A. Govindaraj, C.N.R. Rao, Chem Phys Lett, 418 (2006) 586-590.

[40] X.W. Sun, H.S. Kwok, J Appl Phys, 86 (1999) 408-411.

[41] H.S. Kang, J.S. Kang, J.W. Kim, S.Y. Lee, J Appl Phys, 95 (2004) 1246-1250.

[42] P.F. Carcia, R.S. McLean, M.H. Reilly, G. Nunes, Appl Phys Lett, 82 (2003) 1117-1119.

[43] E.M.C. Fortunato, P.M.C. Barquinha, A.C.M.B.G. Pimentel, A.M.F. Goncalves, A.J.S. Marques, L.M.N. Pereira, R.F.P. Martins, Adv Mater, 17 (2005) 590-+.

[44] B.B. Lakshmi, C.J. Patrissi, C.R. Martin, Chem Mater, 9 (1997) 2544-2550.

[45] M.N. Kamalasanan, S. Chandra, Thin Solid Films, 288 (1996) 112-115.

[46] X.N. Wen, W.Z. Wu, Y. Ding, Z.L. Wang, J Mater Chem, 22 (2012) 9469-9476.

[47] J.X. Wang, X.W. Sun, Y. Yang, H. Huang, Y.C. Lee, O.K. Tan, L. Vayssieres, Nanotechnology, 17 (2006) 4995-4998.

[48] B. Sun, H. Sirringhaus, Nano Lett, 5 (2005) 2408-2413.

[49] S. Xu, Y. Wei, M. Kirkham, J. Liu, W. Mai, D. Davidovic, R.L. Snyder, Z.L. Wang, J Am Chem Soc, 130 
(2008) 14958-+.

[50] S. Strite, H. Morkoc, J Vac Sci Technol B, 10 (1992) 1237-1266.

[51] P.K. Nair, M.T.S. Nair, V.M. Garcia, O.L. Arenas, Y. Pena, A. Castillo, I.T. Ayala, O. Gomezdaza, A. Sanchez, J. Campos, H. Hu, R. Suarez, M.E. Rincon, Sol Energ Mat Sol C, 52 (1998) 313-344.

[52] A. Janotti, C.G. Van de Walle, Phys Rev B, 76 (2007).

[53] W.Z. Wu, Z.L. Wang, Nano Lett, 11 (2011) 2779-2785.

[54] F. Zhang, S.M. Niu, W.X. Guo, G. Zhu, Y. Liu, X.L. Zhang, Z.L. Wang, Acs Nano, 7 (2013) 4537-4544.

[55] Q. Yang, W.H. Wang, S. Xu, Z.L. Wang, Nano Lett, 11 (2011) 4012-4017.

[56] J.Q. Xu, Q.Y. Pan, Y.A. Shun, Z.Z. Tian, Sensor Actuat B-Chem, 66 (2000) 277-279.

[57] H. Morkoç, H. Morkoç, Handbook of nitride semiconductors and devices, Wiley-VCH ; John Wiley distributor, Weinheim

Chichester, 2008.

[58] S.B. Zhang, S.H. Wei, A. Zunger, Phys Rev B, 63 (2001).

[59] D.C. Look, D.C. Reynolds, C.W. Litton, R.L. Jones, D.B. Eason, G. Cantwell, Appl Phys Lett, 81 (2002) 1830-1832.

[60] K.C. Pradel, W.Z. Wu, Y.S. Zhou, X.N. Wen, Y. Ding, Z.L. Wang, Nano Lett, 13 (2013) 2647-2653.

[61] T.M. Barnes, K. Olson, C.A. Wolden, Appl Phys Lett, 86 (2005).

[62] A. Janotti, C.G. Van de Walle, Rep Prog Phys, 72 (2009).

[63] U. Ozgur, Y.I. Alivov, C. Liu, A. Teke, M.A. Reshchikov, S. Dogan, V. Avrutin, S.J. Cho, H. Morkoc, J Appl Phys, 98 (2005).

[64] S. Nakamura, T. Mukai, M. Senoh, Jpn J Appl Phys 2, 30 (1991) L1998-L2001.

[65] S.J. Pearton, F. Ren, A.P. Zhang, K.P. Lee, Mat Sci Eng R, 30 (2000) 55-212.

[66] X.D. Wang, J.H. Song, C.J. Summers, J.H. Ryou, P. Li, R.D. Dupuis, Z.L. Wang, J Phys Chem B, 110 (2006) 7720-7724.

[67] B. Wu, A. Heidelberg, J.J. Boland, Nat Mater, 4 (2005) 525-529.

[68] R. Agrawal, B. Peng, E.E. Gdoutos, H.D. Espinosa, Nano Lett, 8 (2008) 3668-3674.

[69] J.E. Allen, D.E. Perea, E.R. Hemesath, L.J. Lauhon, Adv Mater, 21 (2009) 3067-+.

[70] Z.P. Huang, H. Fang, J. Zhu, Adv Mater, 19 (2007) 744-+.

[71] E.M. Freer, O. Grachev, X.F. Duan, S. Martin, D.P. Stumbo, Nat Nanotechnol, 5 (2010) 525-530.

[72] S. Acharya, A.B. Panda, N. Belman, S. Efrima, Y. Golan, Adv Mater, 18 (2006) 210-+.

[73] Y.F. Hu, Y. Zhang, L. Lin, Y. Ding, G. Zhu, Z.L. Wang, Nano Lett, 12 (2012) 3851-3856.

[74] X.N. Wen, W.Z. Wu, Y. Ding, Z.L. Wang, Adv Mater, 25 (2013) 3371-3379.

[75] G.J. Exarhos, S.K. Sharma, Thin Solid Films, 270 (1995) 27-32.

[76] M.K. Puchert, P.Y. Timbrell, R.N. Lamb, J Vac Sci Technol A, 14 (1996) 2220-2230.

[77] X.N. Wen, W.Z. Wu, Z.L. Wang, Nano Energy, 2 (2013) 1093-1100.

[78] A.K. Geim, K.S. Novoselov, Nat Mater, 6 (2007) 183-191.

[79] B. Radisavljevic, A. Radenovic, J. Brivio, V. Giacometti, A. Kis, Nat Nanotechnol, 6 (2011) 147-150.

[80] K.A.N. Duerloo, M.T. Ong, E.J. Reed, J Phys Chem Lett, 3 (2012) 2871-2876.

[81] Y. Li, K.A.N. Duerloo, E.J. Reed, Nano Lett, 14 (2014) 4299-4305.

[82] W.Z. Wu, Y.G. Wei, Z.L. Wang, Adv Mater, 22 (2010) 4711-+.

[83] A. Sawa, Mater Today, 11 (2008) 28-36.

[84] D.H. Kwon, K.M. Kim, J.H. Jang, J.M. Jeon, M.H. Lee, G.H. Kim, X.S. Li, G.S. Park, B. Lee, S. Han, M. Kim, C.S. Hwang, Nat Nanotechnol, 5 (2010) 148-153.

[85] W.Y. Chang, Y.C. Lai, T.B. Wu, S.F. Wang, F. Chen, M.J. Tsai, Appl Phys Lett, 92 (2008). 
[86] L. Goux, P. Czarnecki, Y.Y. Chen, L. Pantisano, X.P. Wang, R. Degraeve, B. Govoreanu, M. Jurczak, D.J. Wouters, L. Altimime, Appl Phys Lett, 97 (2010).

[87] H.X. Li, X.P. Chen, Q. Chen, Q.N. Mao, J.H. Xi, Z.G. Ji, Acta Phys Sin-Ch Ed, 62 (2013).

[88] J.H. Hur, M.J. Lee, C.B. Lee, Y.B. Kim, C.J. Kim, Phys Rev B, 82 (2010).

[89] Q. Yang, Y. Liu, C.F. Pan, J. Chen, X.N. Wen, Z.L. Wang, Nano Lett, 13 (2013) 607-613.

[90] Y. Cui, Q.Q. Wei, H.K. Park, C.M. Lieber, Science, 293 (2001) 1289-1292.

[91] Z.Y. Fan, D.W. Wang, P.C. Chang, W.Y. Tseng, J.G. Lu, Appl Phys Lett, 85 (2004) 5923-5925.

[92] P. Mitra, A.P. Chatterjee, H.S. Maiti, Mater Lett, 35 (1998) 33-38.

[93] R.M. Yu, C.F. Pan, J. Chen, G. Zhu, Z.L. Wang, Adv Funct Mater, 23 (2013) 5868-5874.

[94] C.F. Pan, R.M. Yu, S.M. Niu, G. Zhu, Z.L. Wang, Acs Nano, 7 (2013) 1803-1810.

[95] W.Z. Wu, X.N. Wen, Z.L. Wang, Science, 340 (2013) 952-957.

[96] C.F. Pan, L. Dong, G. Zhu, S.M. Niu, R.M. Yu, Q. Yang, Y. Liu, Z.L. Wang, Nat Photonics, 7 (2013) 752-758.

[97] S.A. Mascaro, H.H. Asada, leee T Robotic Autom, 17 (2001) 698-708.

[98] Z.L. Wang, Adv Mater, 24 (2012) 4630-4631.

[99] S.M. Niu, Y.F. Hu, X.N. Wen, Y.S. Zhou, F. Zhang, L. Lin, S.H. Wang, Z.L. Wang, Adv Mater, 25 (2013) 3701-3706. 
Figure 1: Schematic of the energy band diagram illustrating the piezotronic effect on M-S interface. (a) Negative polarization charges are induced at the interface, increasing the barrier height. (b) Positive polarization charges are induced at the interface, decreasing the barrier height. For the color gradient, red represents positive potential and blue represents negative potential. Reproduced from [98] with permission. Copyright 2012 WILEY-VCH Verlag GmbH \& Co. KGaA, Weinheim.

Figure 2: Schematic of the energy band diagram illustrating the piezotronic effect on p-n junction interface. (a) Positive polarization charges are induced at the interface, creating a dip in the band diagram. (b) Negative polarization charges are induced at the interface, creating a bump in the band diagram. For the color gradient, red represents positive potential and blue represents negative potential. Reproduced from [98] with permission. Copyright 2012 WILEY-VCH Verlag GmbH \& Co. KGaA, Weinheim.

Figure 3: Piezotronic characteristics for devices with low conductivity at the temperature of (a) $300 \mathrm{~K}$, (b) $200 \mathrm{~K}$ and (c) $77 \mathrm{~K}$. Insets of (a) and (b) are magnified plots in the negative voltage range. Reproduced from [31] with permission. Copyright 2013 American Chemical Society.

Figure 4: Piezotronic characteristics for devices with (a) low, (b) moderate and (c) high conductivity at the temperature of $77 \mathrm{~K}$. Reproduced from [31] with permission. Copyright 2013 American Chemical Society.

Figure 5: Schematic of energy band diagram illustrating the piezotronic effect of p-type $\mathrm{ZnO}$ at M-S interface. (a) No strain is applied. (b) Tensile strain is applied, inducing positive charges on the right and negative charges on the left. (c) Compressive strain is applied, inducing negative charges on the right and positive charges on the left. Color gradient indicates the distribution of piezopotential, with red for positive and blue for negative potentials. Reproduced from [60] with permission. Copyright 2013 American Chemical Society.

Figure 6: (a) Characteristics of the p-type $\mathrm{ZnO} \mathrm{NW}$ based FET. Inset is the SEM image of the device. (a) Characteristics of the p-type $\mathrm{ZnO} \mathrm{NW}$ based piezotronic transistor. Inset shows the change in barrier height and current as a function of applied strain. Reproduced from [60] with permission. Copyright 2013 American Chemical Society.

Figure 7: (a) TEM, electron diffraction and high resolution TEM images of the RF sputtered $\mathrm{ZnO}$ thin film. (b) Piezotronic characteristics of the $\mathrm{ZnO}$ thin film based device. Reproduced from [74] with permission. Copyright 2013 WILEY-VCH Verlag GmbH \& Co. KGaA, Weinheim. 
Figure 8: Piezotronic characteristics of the device under three different source-drain bias voltage. Tensile strain is defined as logic input " 1 " and compressive strain is defined as logic input "0". Reproduced from [82] with permission. Copyright 2010 WILEY-VCH Verlag GmbH \& Co. KGaA, Weinheim.

Figure 9: (a) Schematics of the structures and symbols of a strain-gated logic inverter. (b) The strain-voltage transfer diagram of the inverter. Inset is the logic table and an optical image of the inverter. Reproduced from [82] with permission. Copyright 2010 WILEY-VCH Verlag GmbH \& Co. KGaA, Weinheim.

Figure 10: (a) Resistive memory switching performance under $0 \%$ strain, $1.17 \%$ tensile strain and $0.76 \%$ compressive strain. (b) Dependence of threshold voltages on strain. The red curve corresponds to the source side and the black curve corresponds to the drain side. Reproduced from [53] with permission. Copyright 2011 American Chemical Society.

Figure 11: Schematic of the device under (a) zero strain, (b) compressive strain and (c) tensile strain. The color gradient represents the distribution of piezopotential with red for positive and blue for negative potentials. (d) Piezotronic characteristics of the device under various strain levels. (e) Magnification of the characteristics for the negative voltage range. (f) Dependence of the relative current change on applied strain. (g) Dependence of the relative emission intensity and external efficiency on applied strain. (h) CCD images recording the emission strength variation of the LED under different strains. Reproduced from [89] with permission. Copyright 2013 American Chemical Society.

Figure 12: (a) Schematic of the thin film solar cell under test. (b) Change of solar cell performance under applied strain for the $\mathrm{ZnO}$ thin film before optimization. (c) Change of solar cell performance under applied strain for the $\mathrm{ZnO}$ thin film after optimization. Reproduced from [77] with permission. Copyright 20132014 Elsevier B.V..

Figure 13: Piezotronic effect on the sensitivity of the room-temperature oxygen sensor. (a) 3-D scatter plot of the sensor current at $1 \mathrm{~V}$ bias under different strain and oxygen partial pressure. (b) Relative current change of the sensor at $1 \mathrm{~V}$ bias under different strain and oxygen partial pressure. (c) 3-D scatter plot of the sensor current at $-1 \mathrm{~V}$ bias under different strain and oxygen partial pressure. (d) Relative current change of the sensor at $-1 \mathrm{~V}$ bias under different strain and oxygen partial pressure. Reproduced from [99] with permission. Copyright 2013 WILEY-VCH Verlag GmbH \& Co. KGaA, Weinheim. 
Figure 14: (a) Schematic of the device design eliminating the gate electrode and schematic of the matrix structure. (b) Equivalent circuit of the matrix device. (c) Optical image of the transparent device laying on a piece of color printed paper. Reproduced from [95] with permission. Copy right 2013 American Association for the Advancement of Science.

Figure 15: (a) Optical images obtained at different fabrication steps of the device. (b) SEM image of the as-synthesized $\mathrm{ZnO} \mathrm{NWs}$ array. The inset is the image for one pixel. Reproduced from [95] with permission. Copy right 2013 American Association for the Advancement of Science.

Figure 16: (a) Topological image of the selected 23 pixels and their corresponding current without and with localized strain. (b) Current of the selected 23 pixels over a period of time. Time sessions marked with blue background are the ones with localized strain. (c) Current variation of pixel 46 over time under varying applied pressure. Top-right inset summarizes the relationship between applied pressure and current change. Reproduced from [95] with permission. Copy right 2013 American Association for the Advancement of Science.

Figure 17: (a) Distribution of the initial current of all pixels under zero strain. (b) Current contour plot demonstrating the pressure imaging ability of the device. (c) Multidimensional pressure imaging, demonstrating the device's ability to record both the position as well as strength of applied pressure. Reproduced from [95] with permission. Copy right 2013 American Association for the Advancement of Science.

Figure 18: (a) Schematic of the device structure and experimental approach. (b) SEM image of the as-grown $\mathrm{ZnO} \mathrm{NWs}$ array on GaN substrate. (c) Optical image of the LED array lit up. (d) The emission profile and emission image of five selected LEDs. (e) Dynamic response of the LED current under periodical strain. The inset shows one response cycle, illustration the fast response and reset time. (f) Relationship between the enhancement of emission intensity and the applied strain. Reproduced from [96] with permission. Copy right 2013 Macmillan Publishers Limited.

Figure 19: (a) Illustration of the experimental approach. (b) Optical image of the convex mold. (c)-(e) Electroluminescence images of the device at the strain of $0 \%$, $-0.06 \%$ and $-0.15 \%$. (f) Electroluminescence contour image showing the pressure imaging ability of the device. (g) Emission intensity enhancement of the LEDs marked with pink dashed line in (e) under $-0.06 \%$ and $-0.15 \%$ strain respectively. (h) Emission intensity perturbation of the LEDs marked with red dashed line in (e) under $-0.06 \%$ and $-0.15 \%$ strain respectively. Reproduced from [96] with permission. Copy right 2013 Macmillan Publishers Limited.

Figure 20: Schematics showing that with piezoelectric semiconductors as the material 
foundation, the field of piezoelectricity, electronics, photonics and chemistry can be actively linked together, which then can give rise to a large number of significant applications. 
(a)

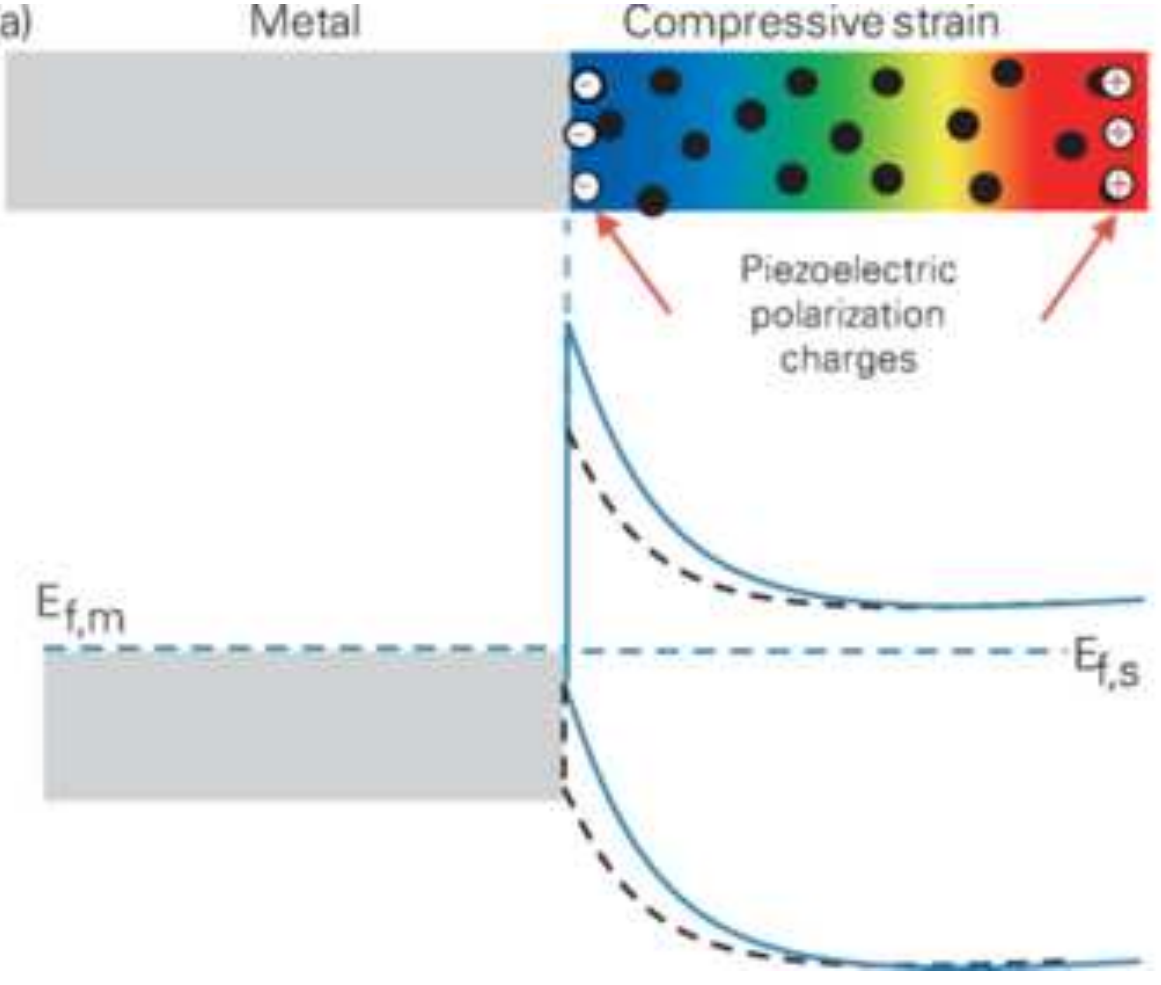

(b)

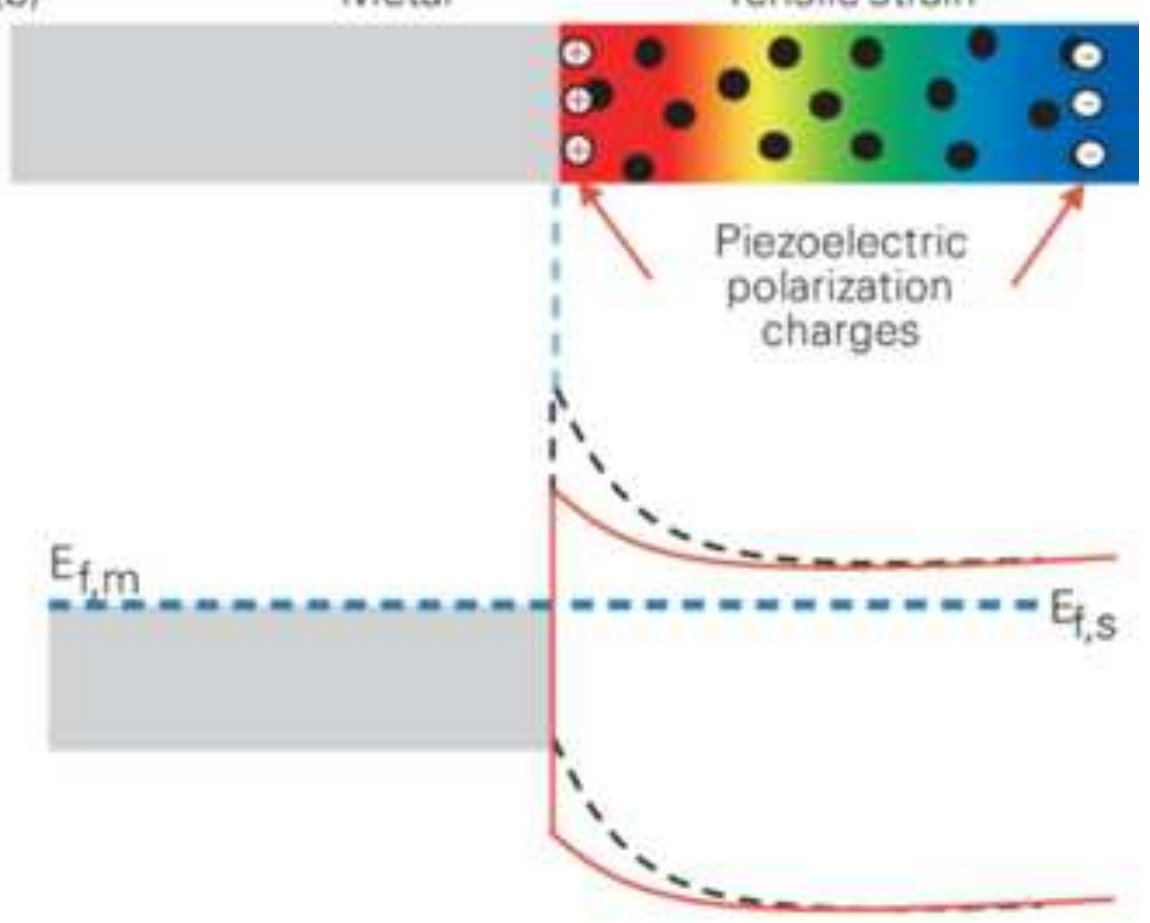



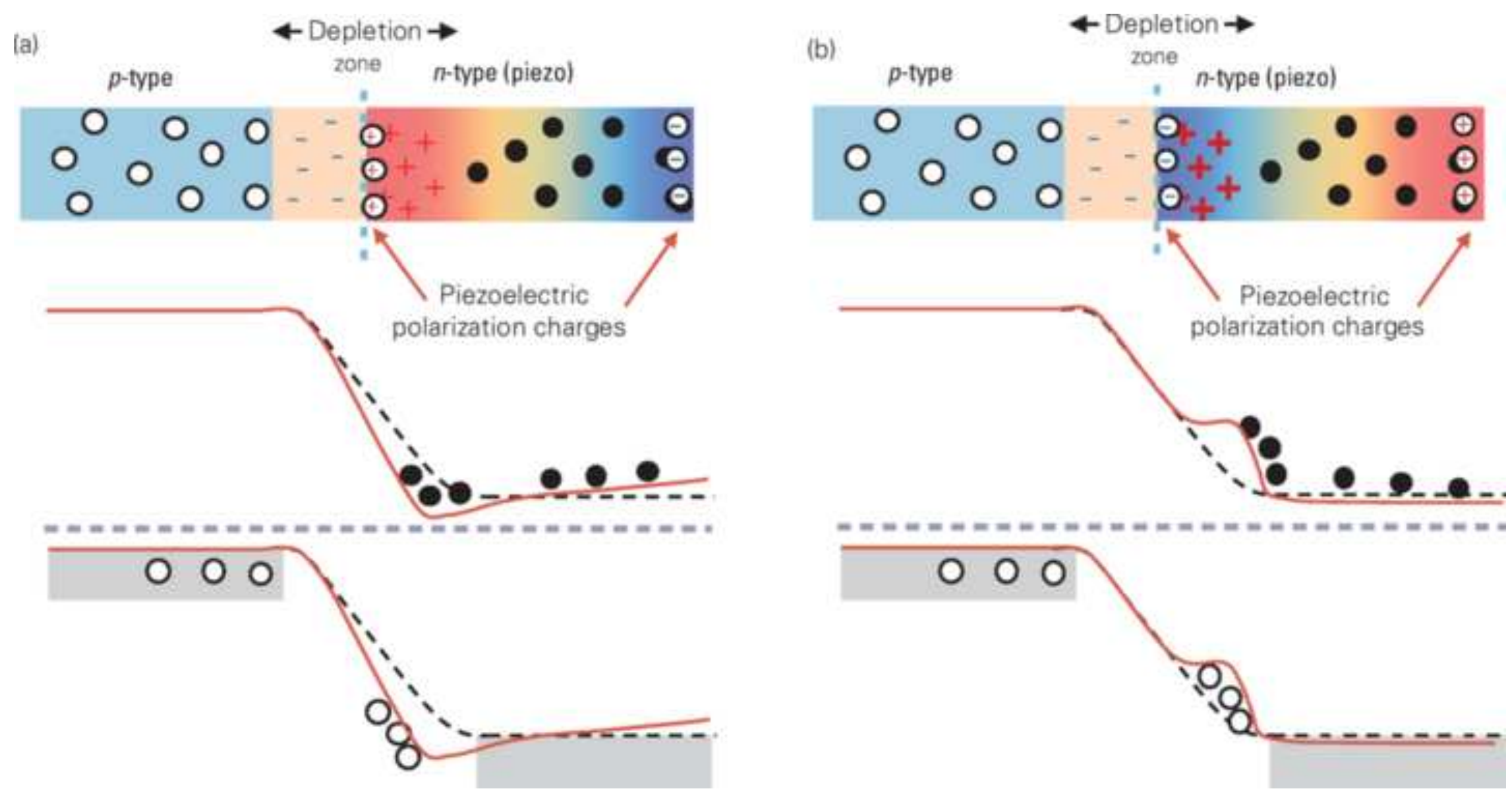

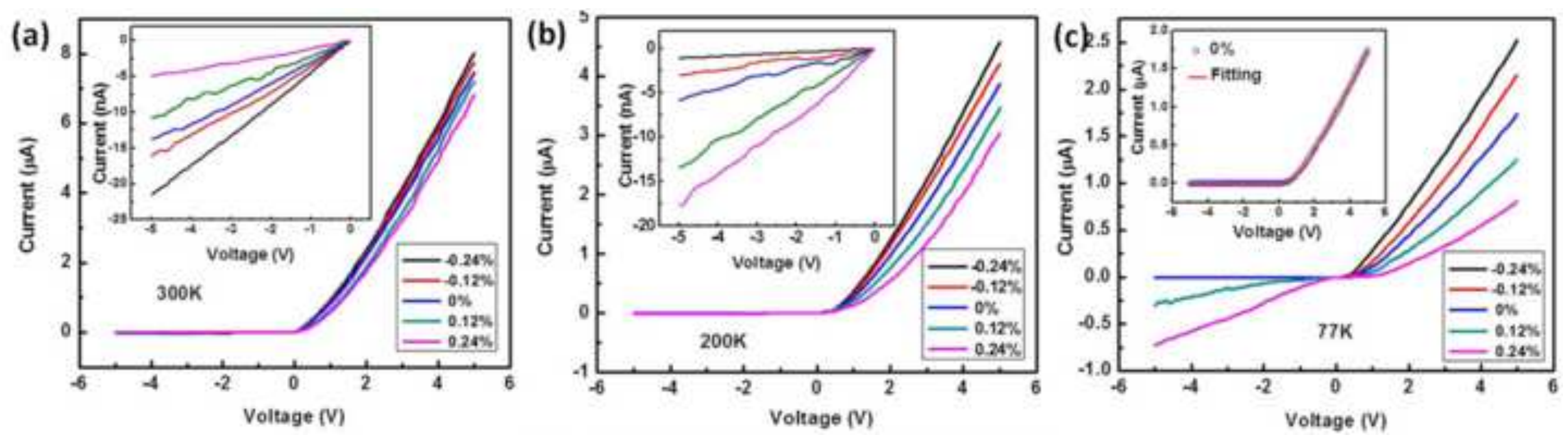

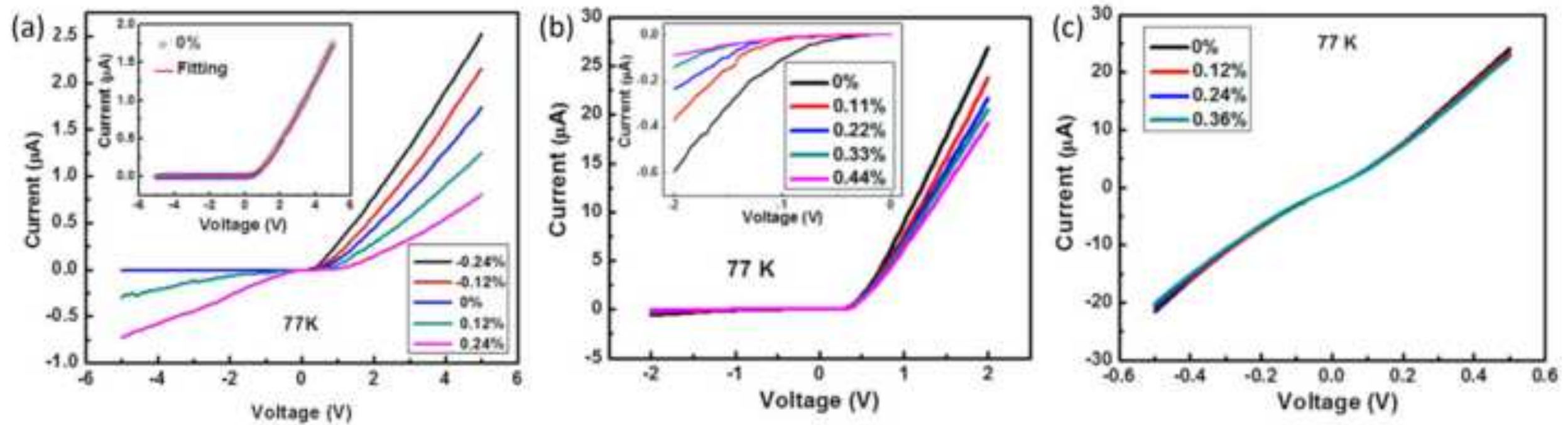
Unstrained

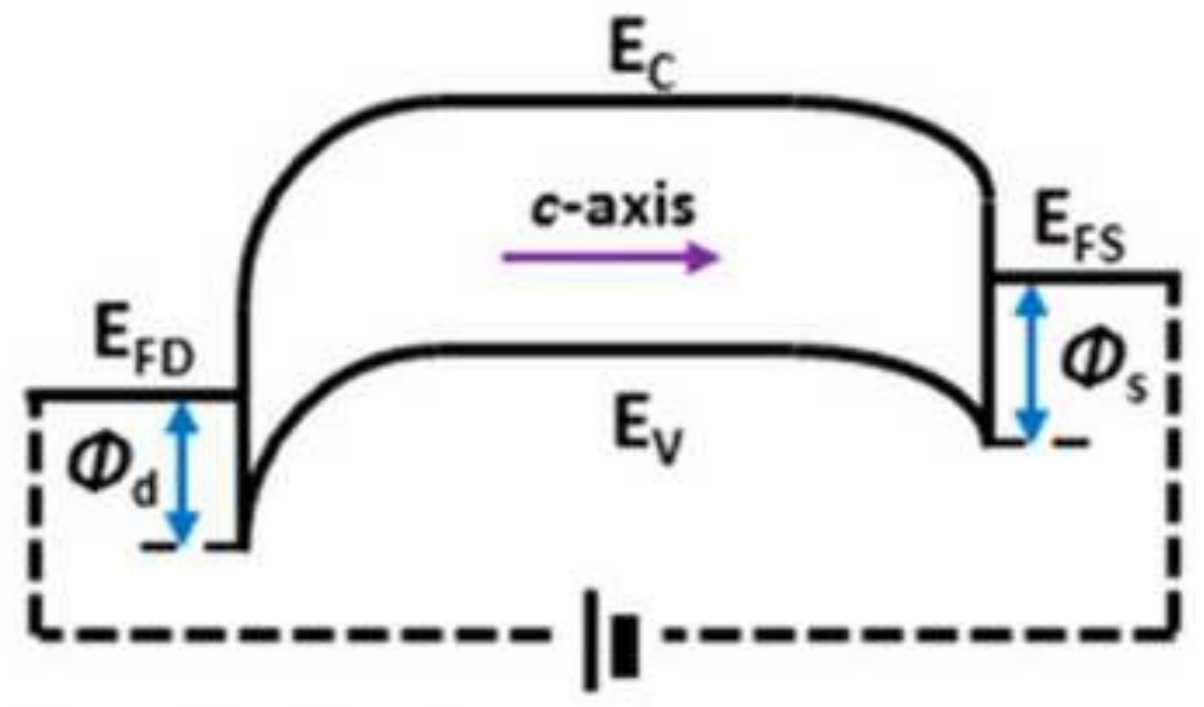

Tensile Strain

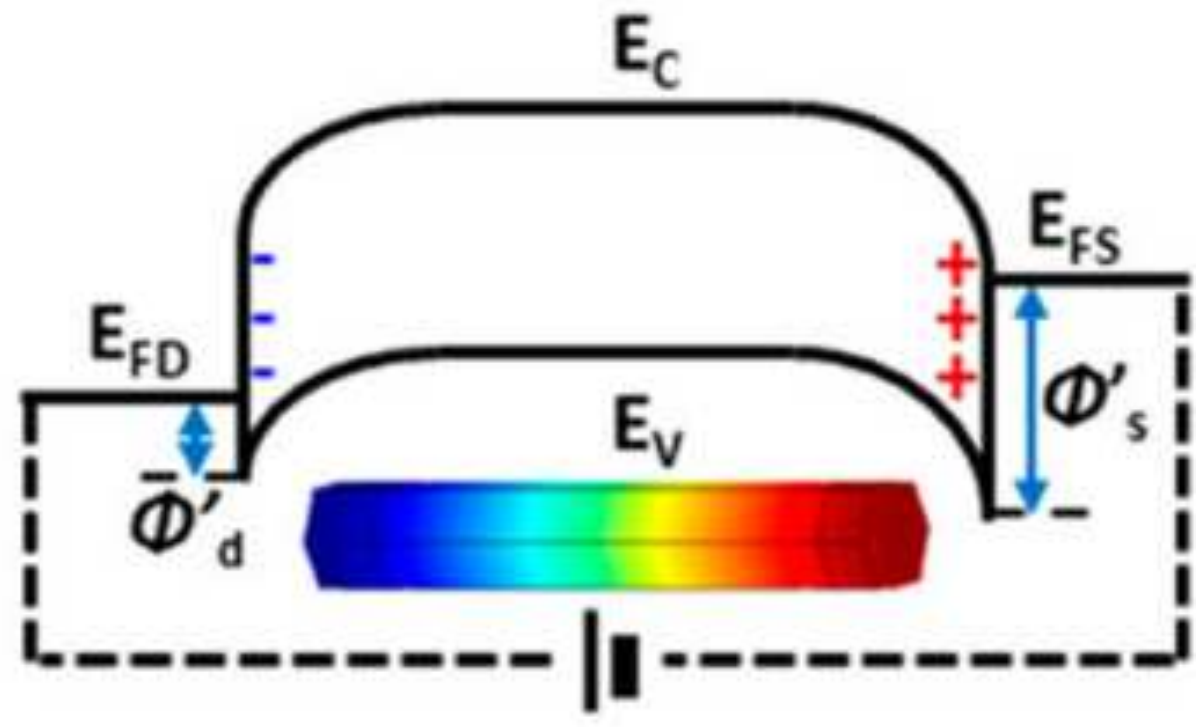

Compressive strain

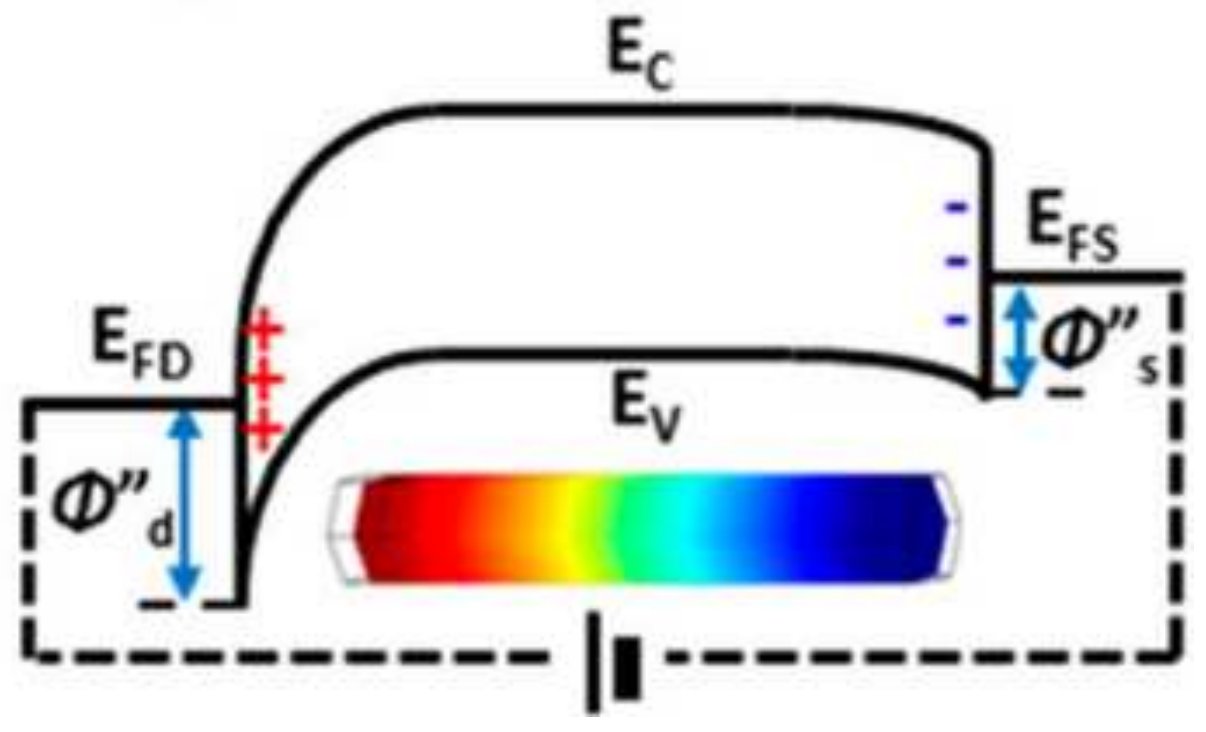



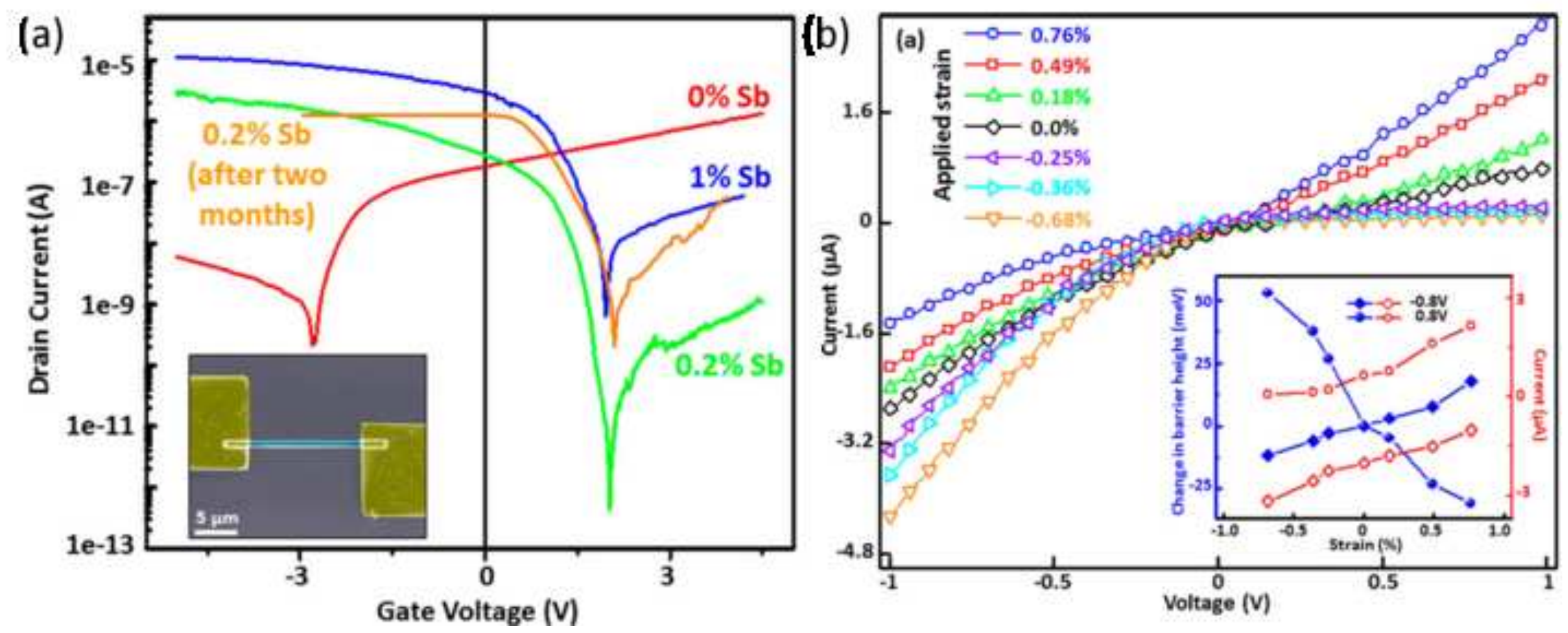

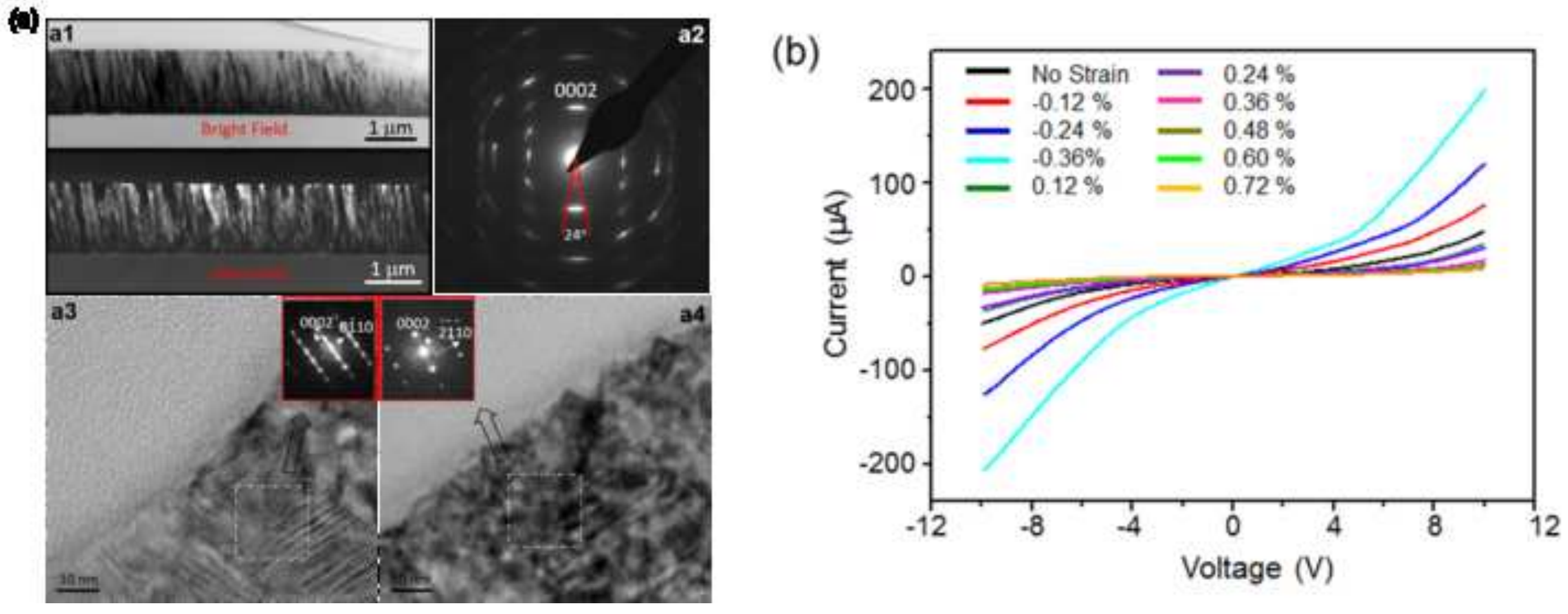


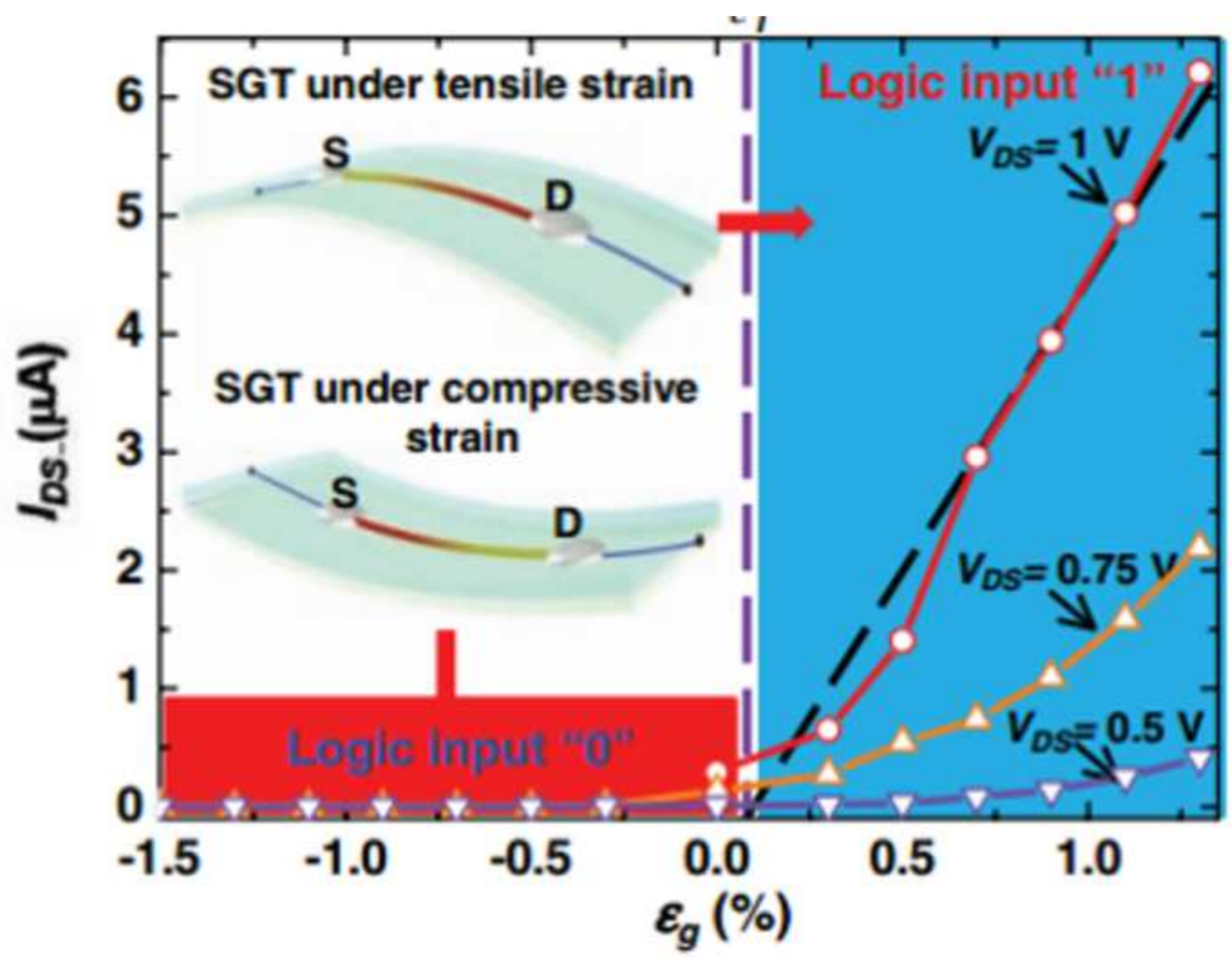




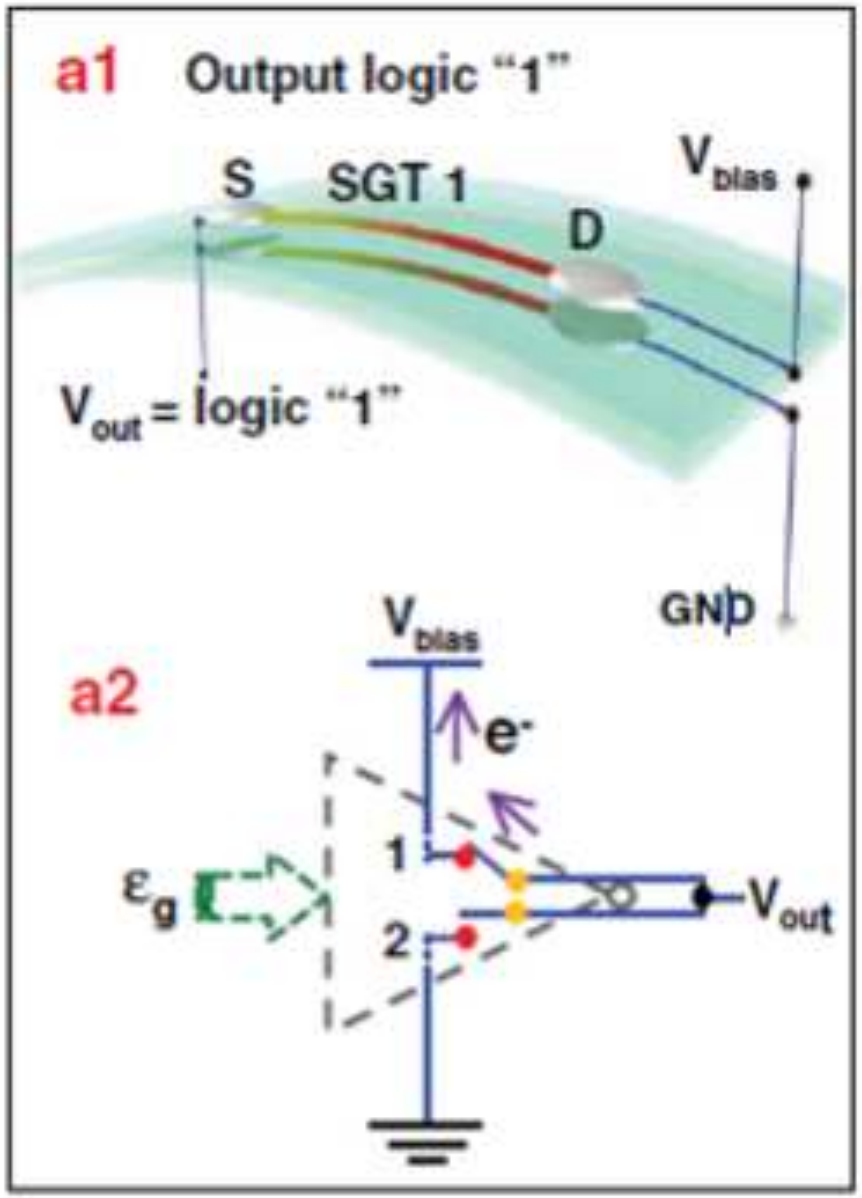

b Strain applied to SGT2 for output logic "1" a3 Ouput logic "0"

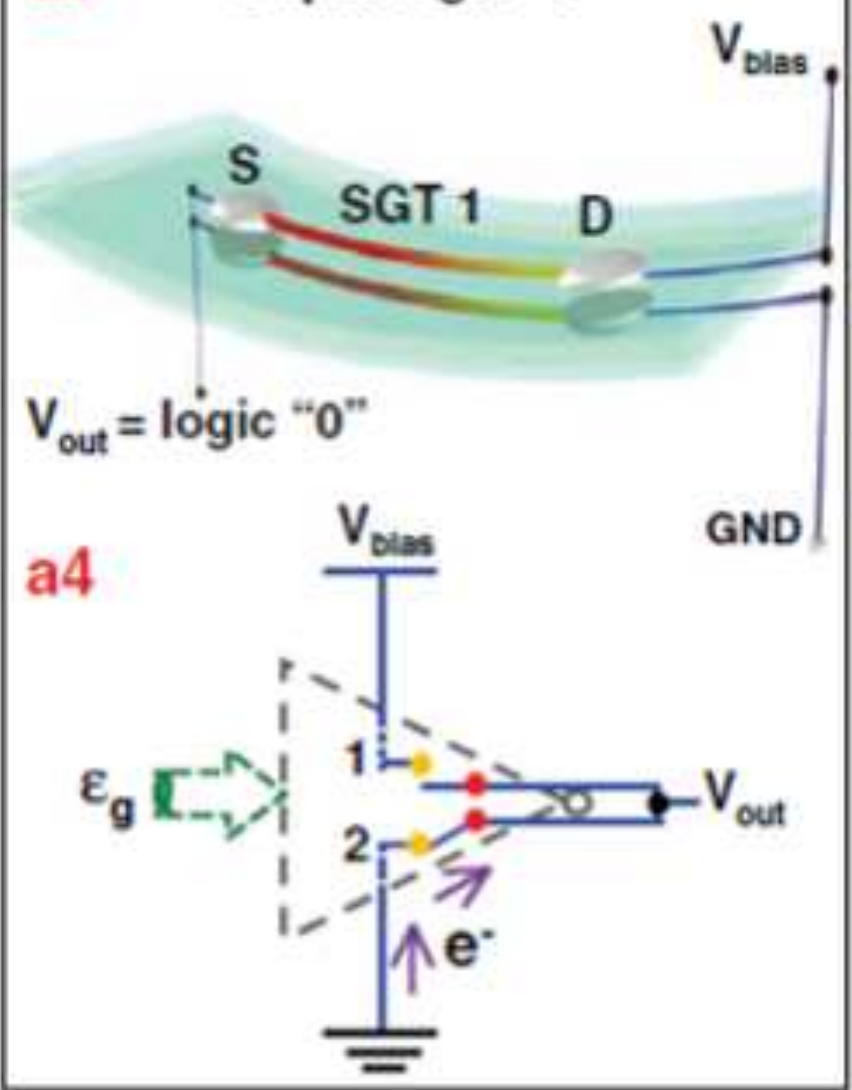

Strain applied to SGT2

for output logic "0"

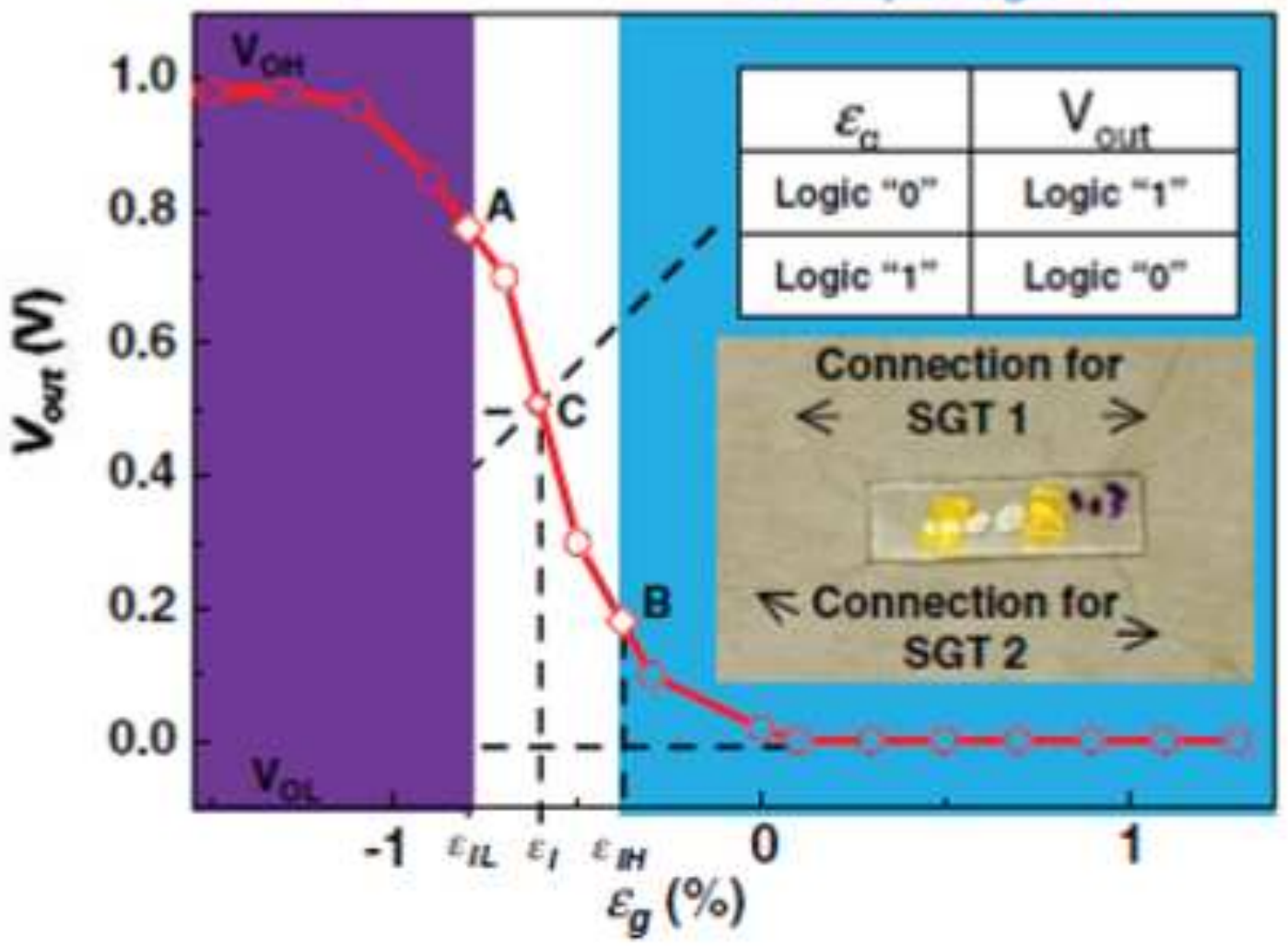




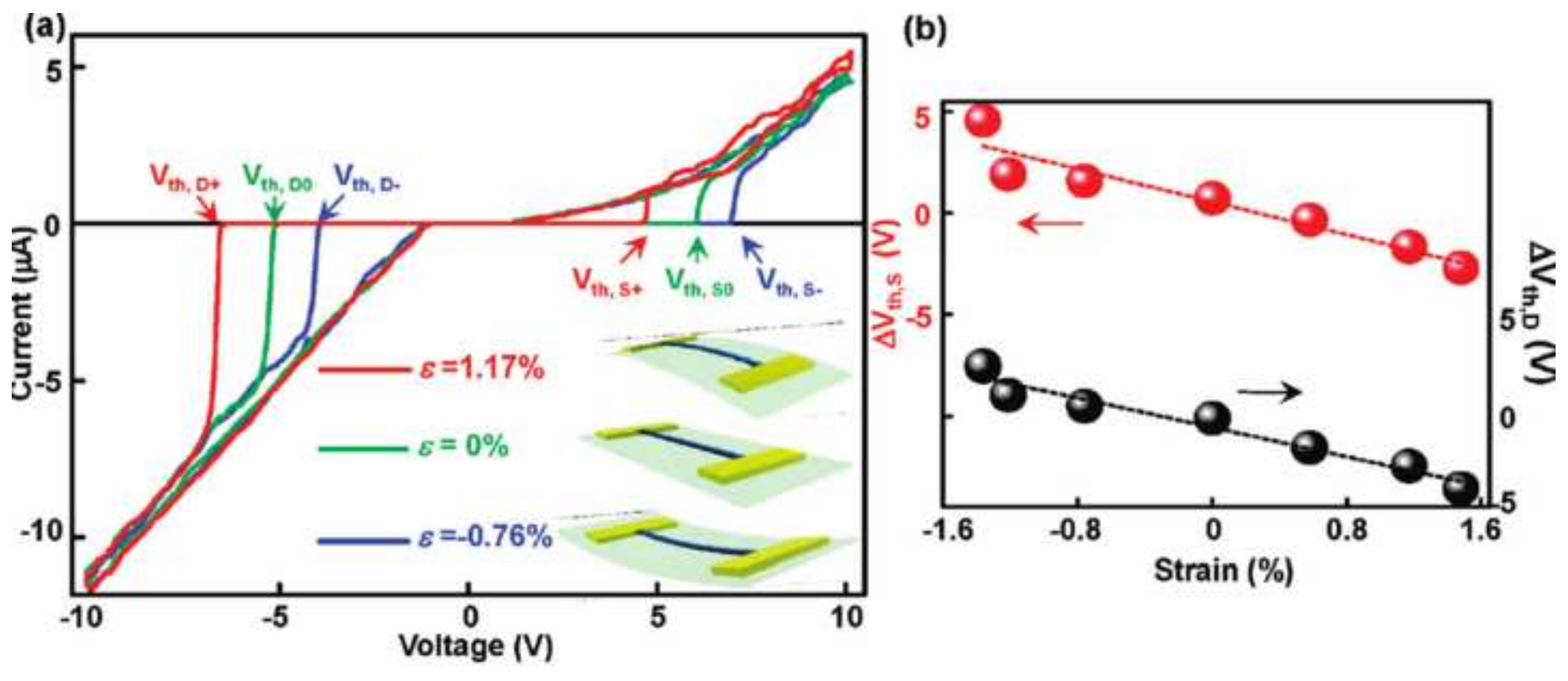


(a)
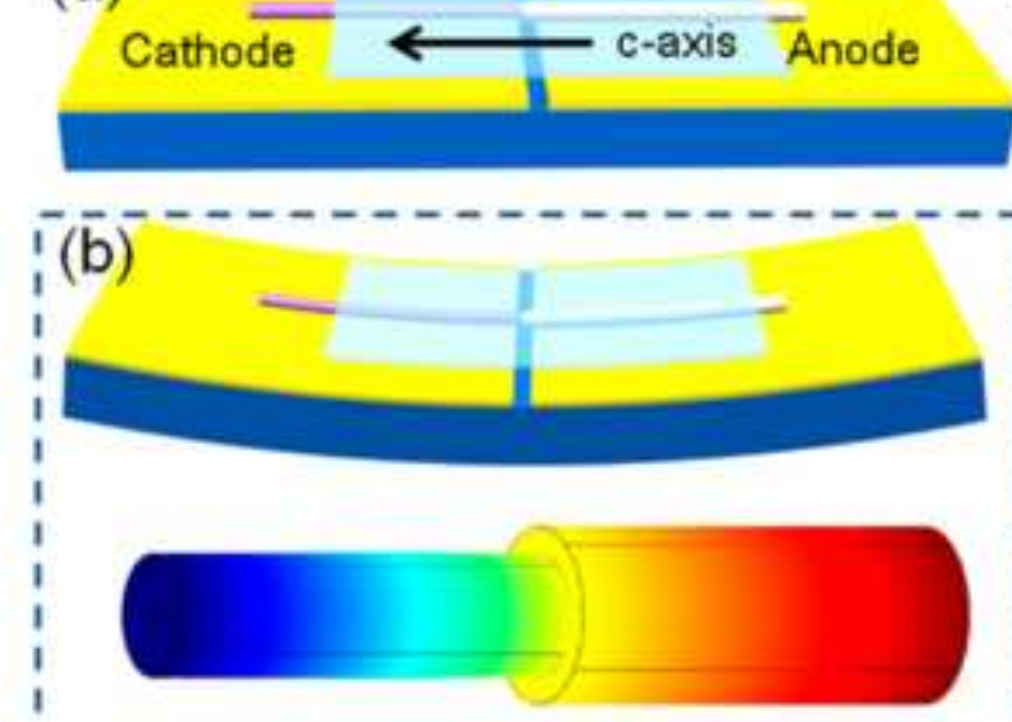

(c)
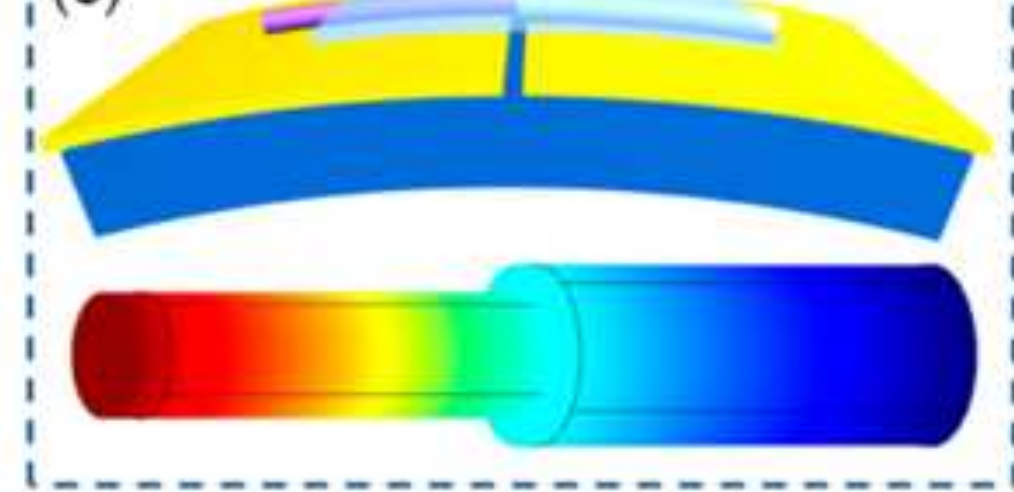

(d)

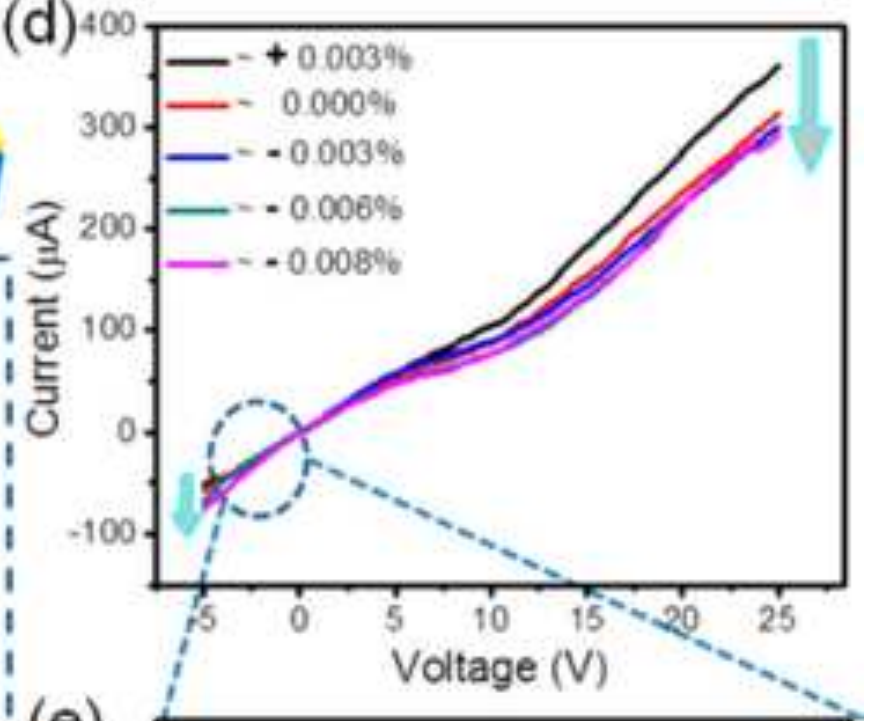

(e)

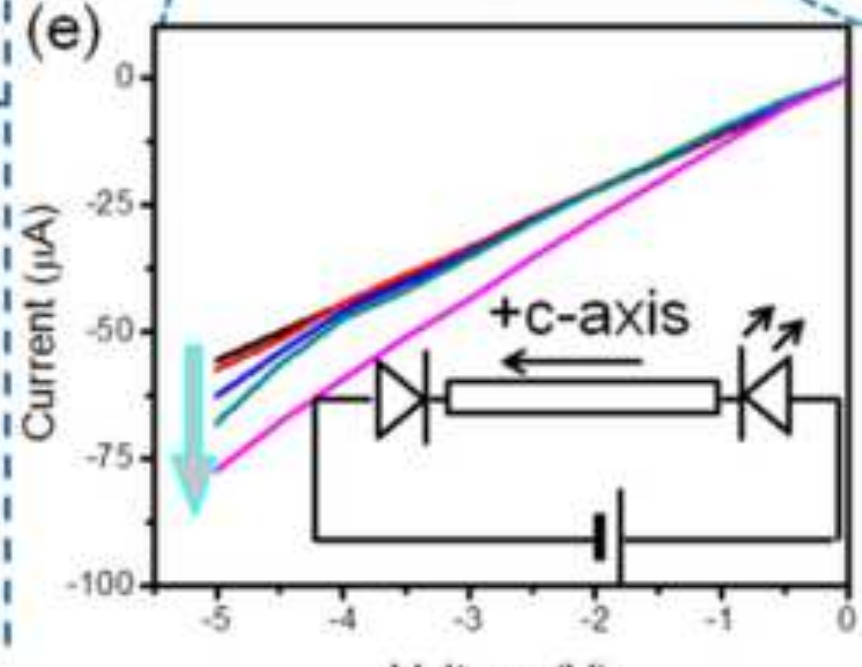

Voltage (V)
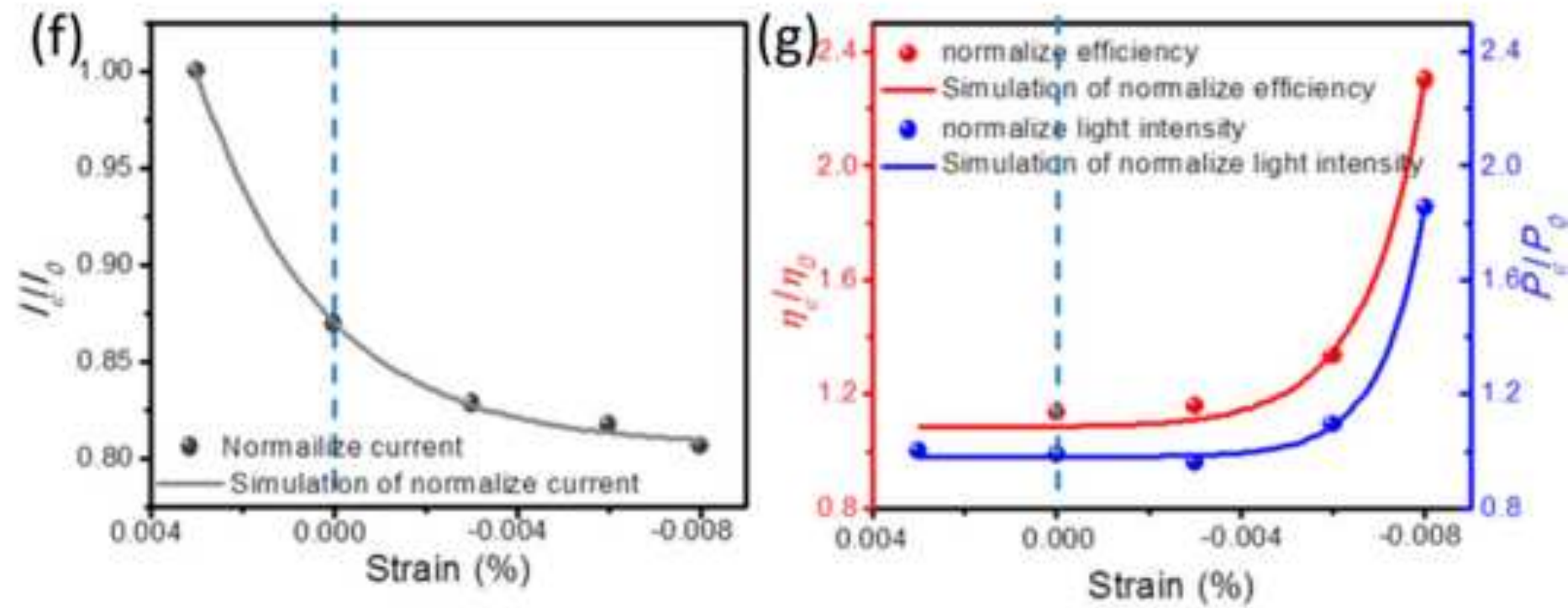

(h)
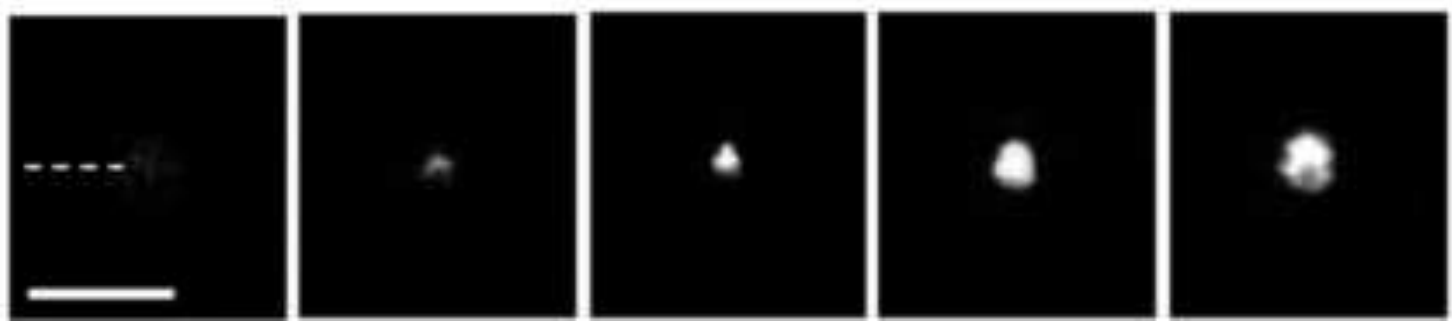
(a)

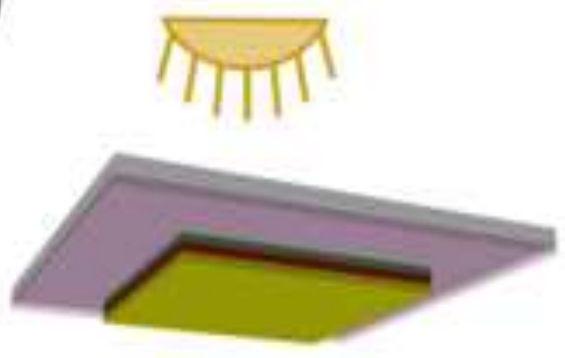

플

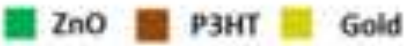

(b)

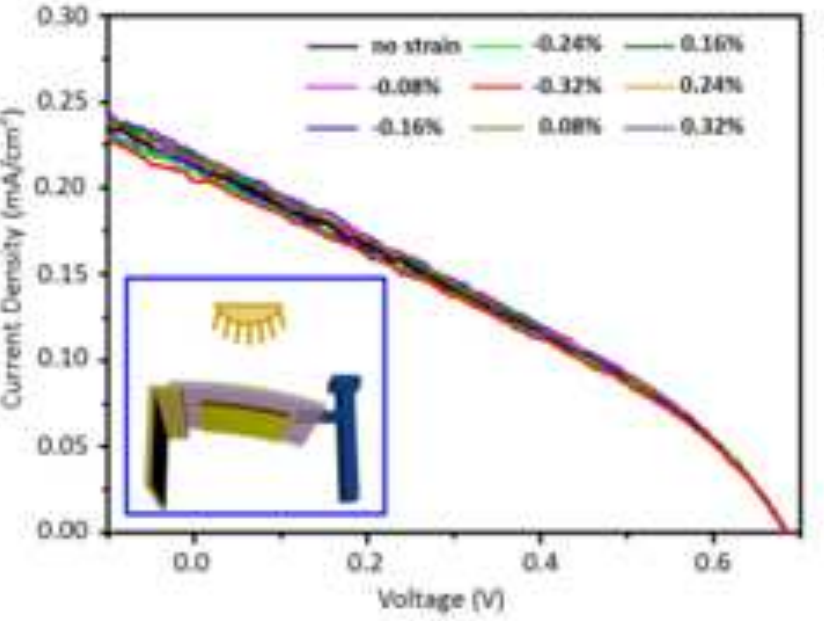

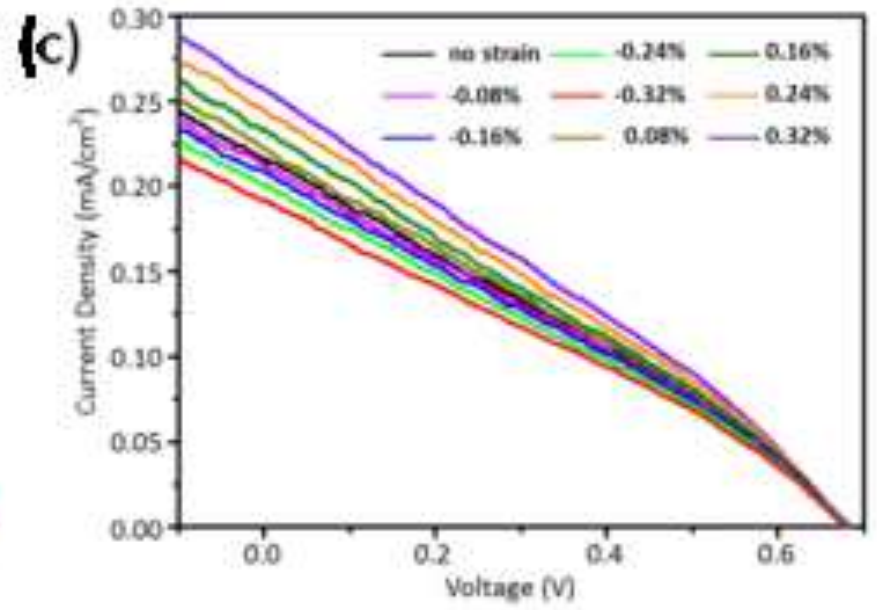


(a)

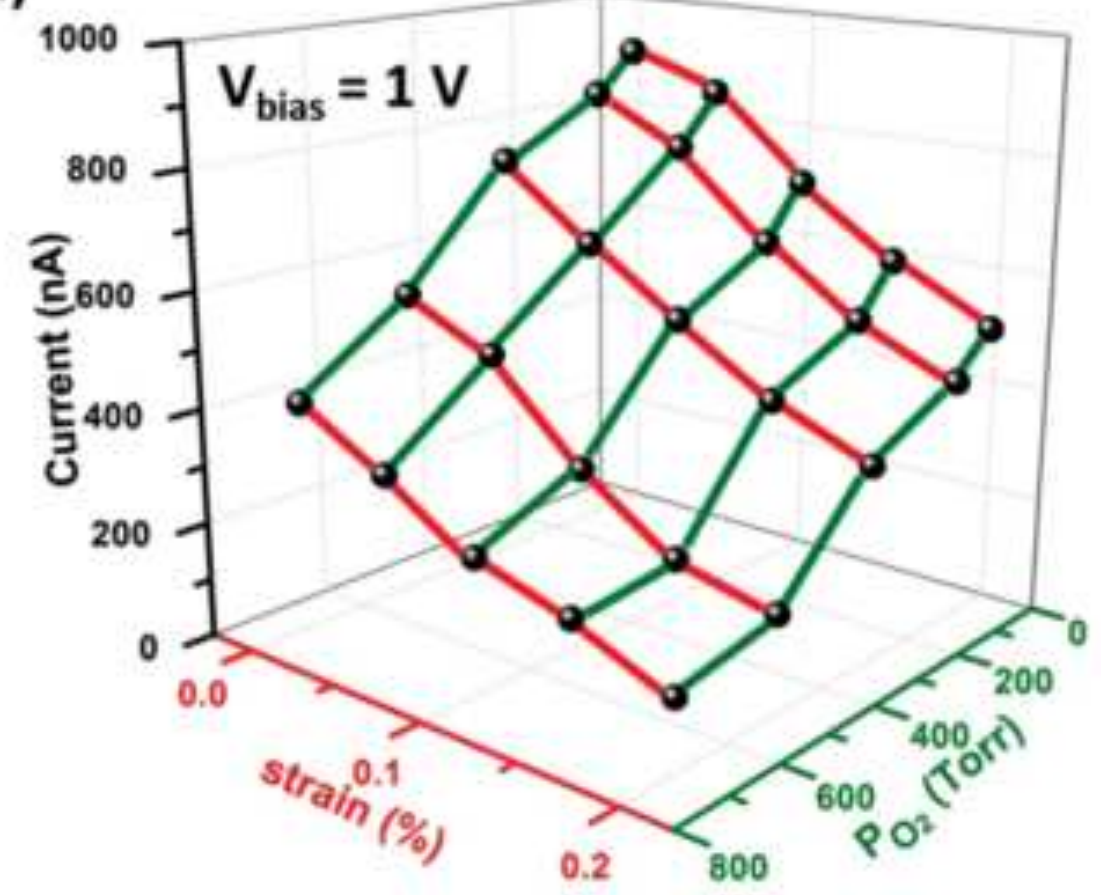

(c)

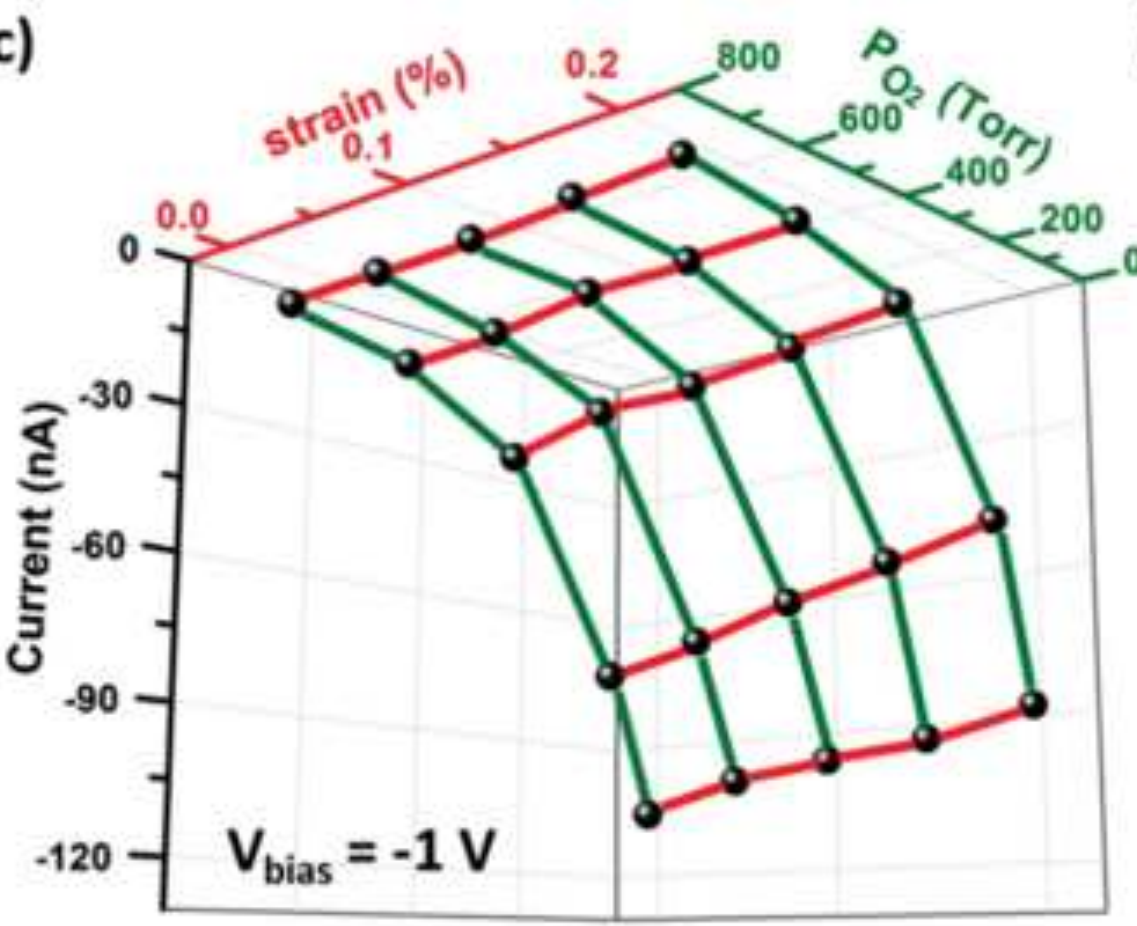

(b)

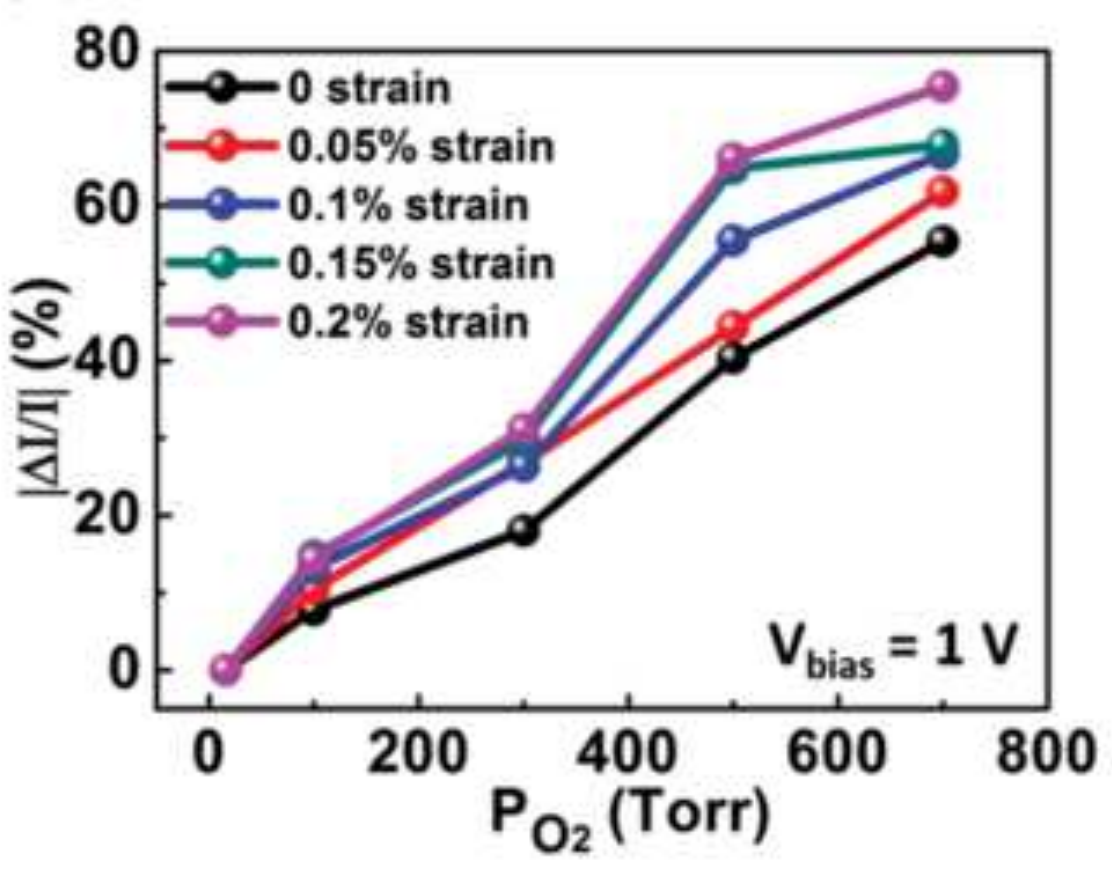

(d)

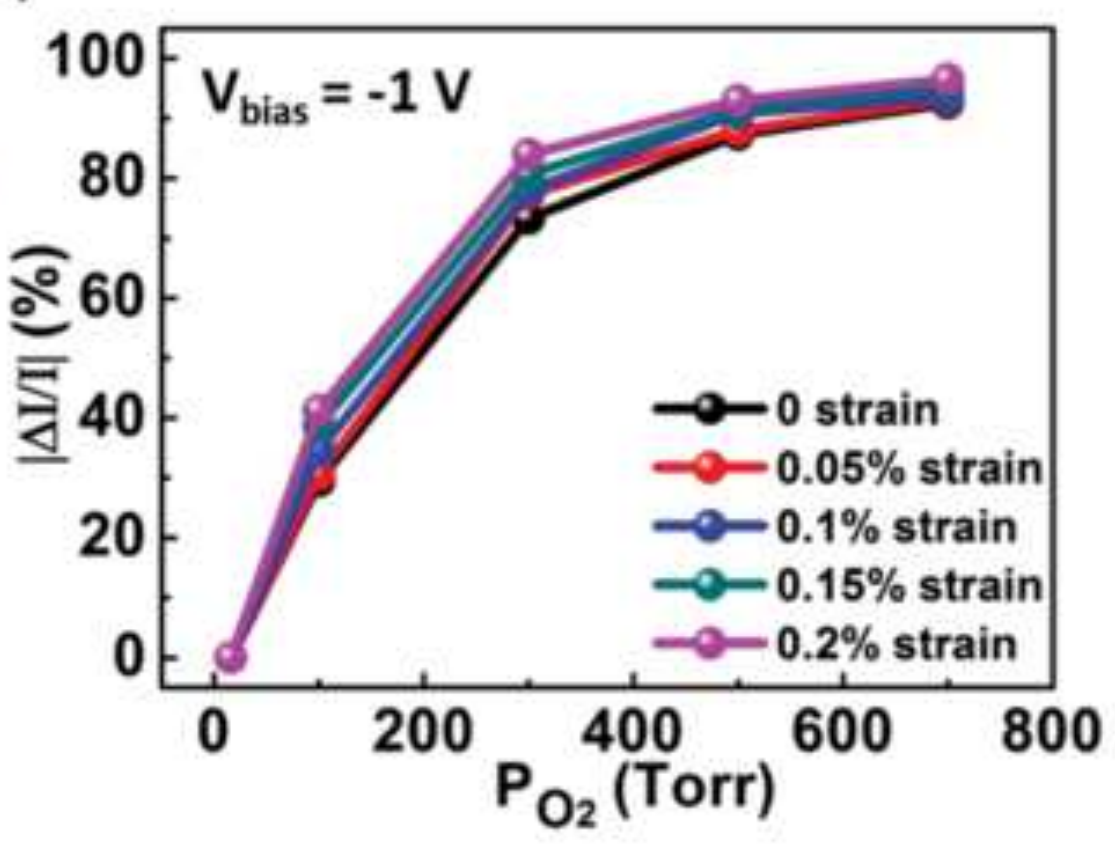



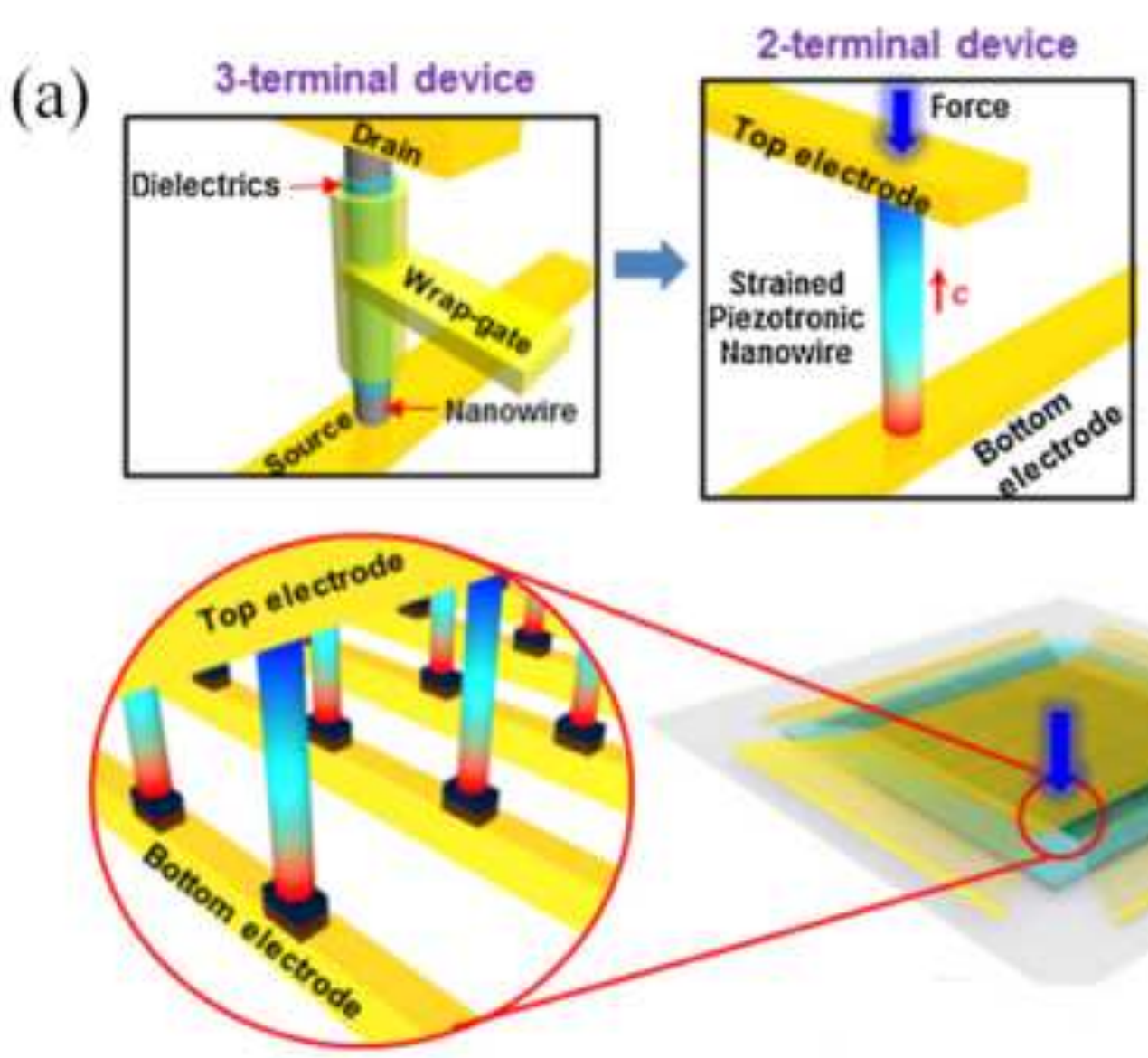

(b)

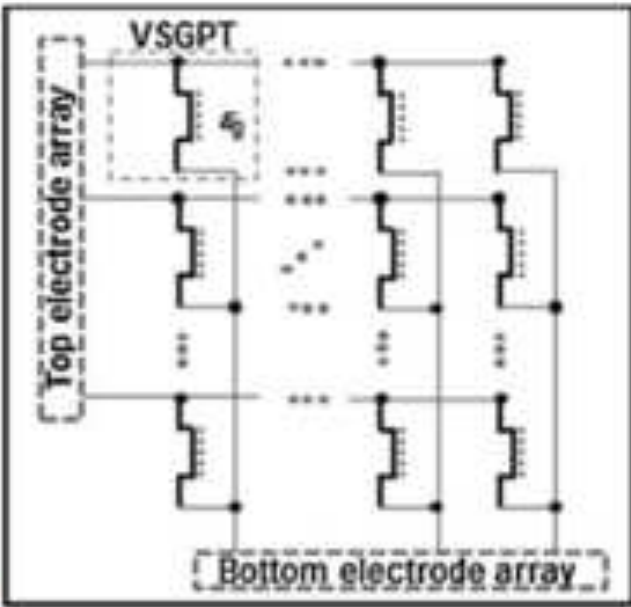

(c)

Piezotronics - Large-Scale' Integration

$0.5 \mathrm{~mm}$ 
Figure 15

Click here to download high resolution image
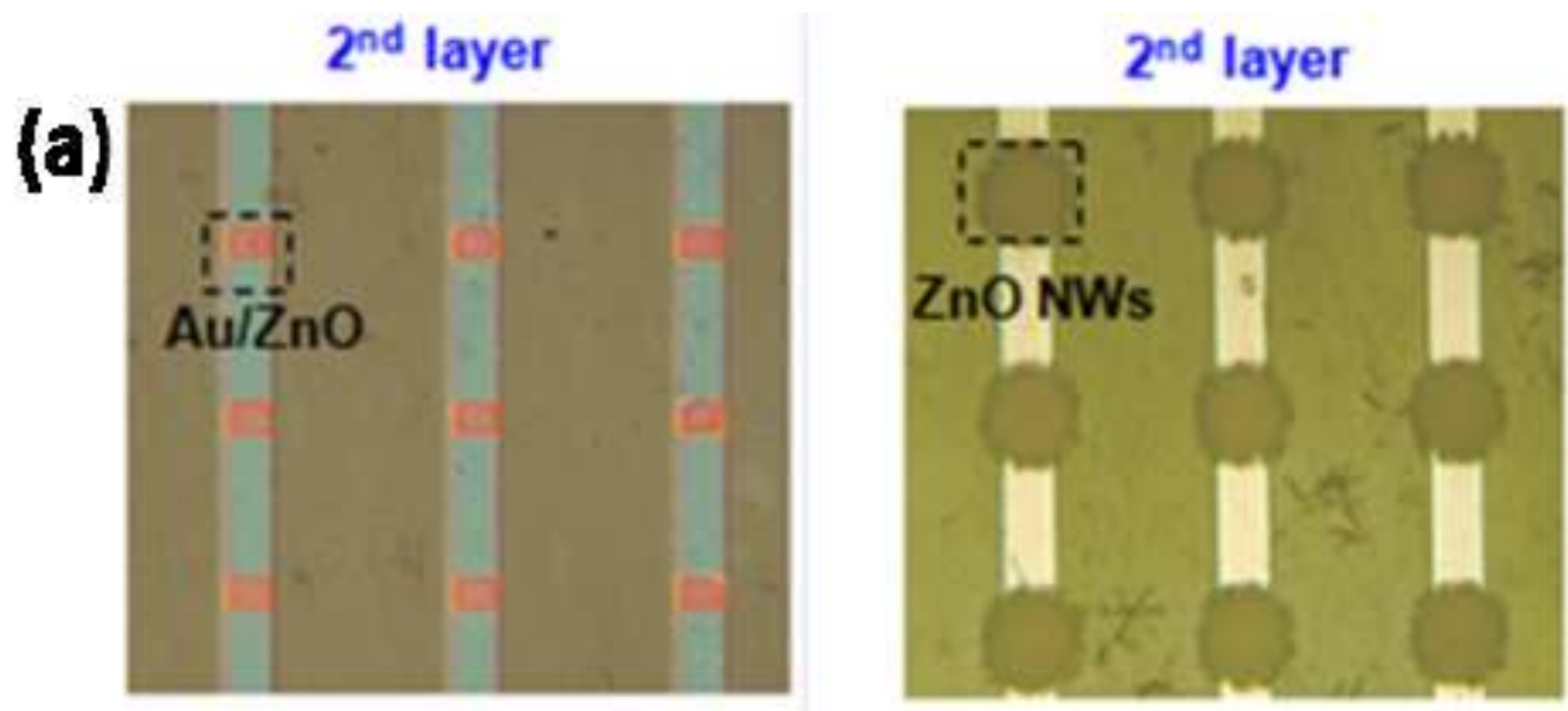

\section{$2^{\text {nd }}$ layer}

SU 8

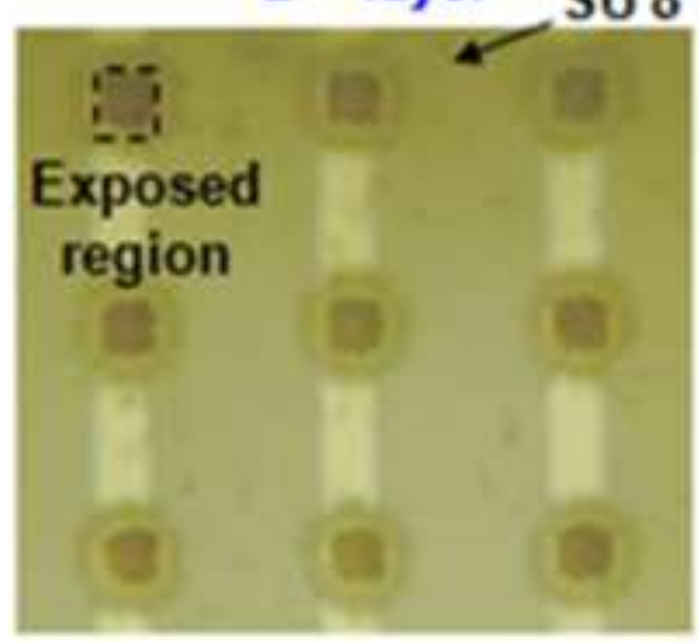

$10 \mathrm{\mu m}$

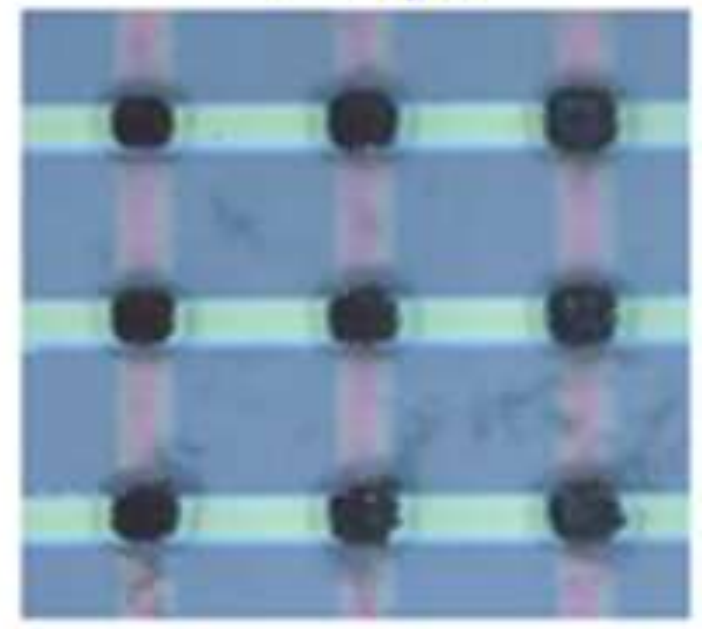


(a) $\underbrace{}_{100 \mathrm{~m}}$

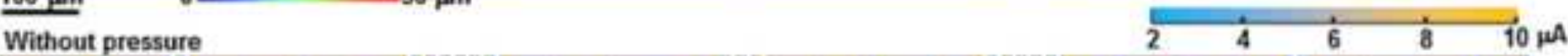

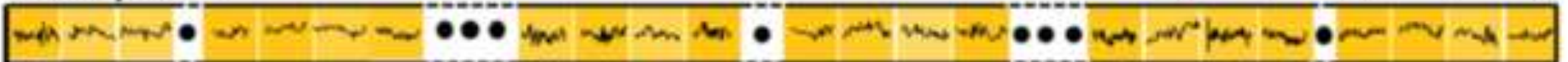
With local pressure around pixels 45 and $45 \quad 4 \quad 8$
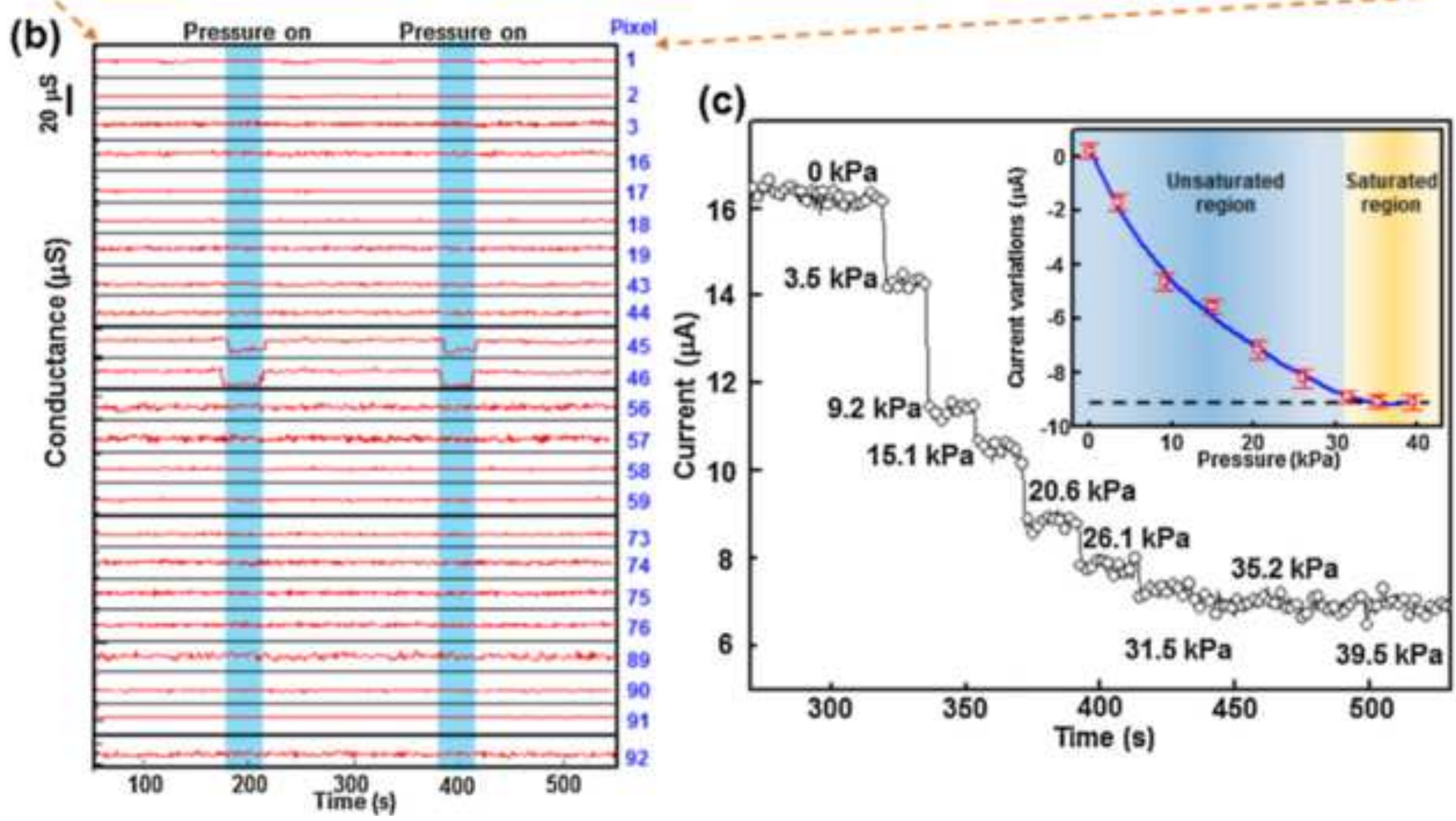

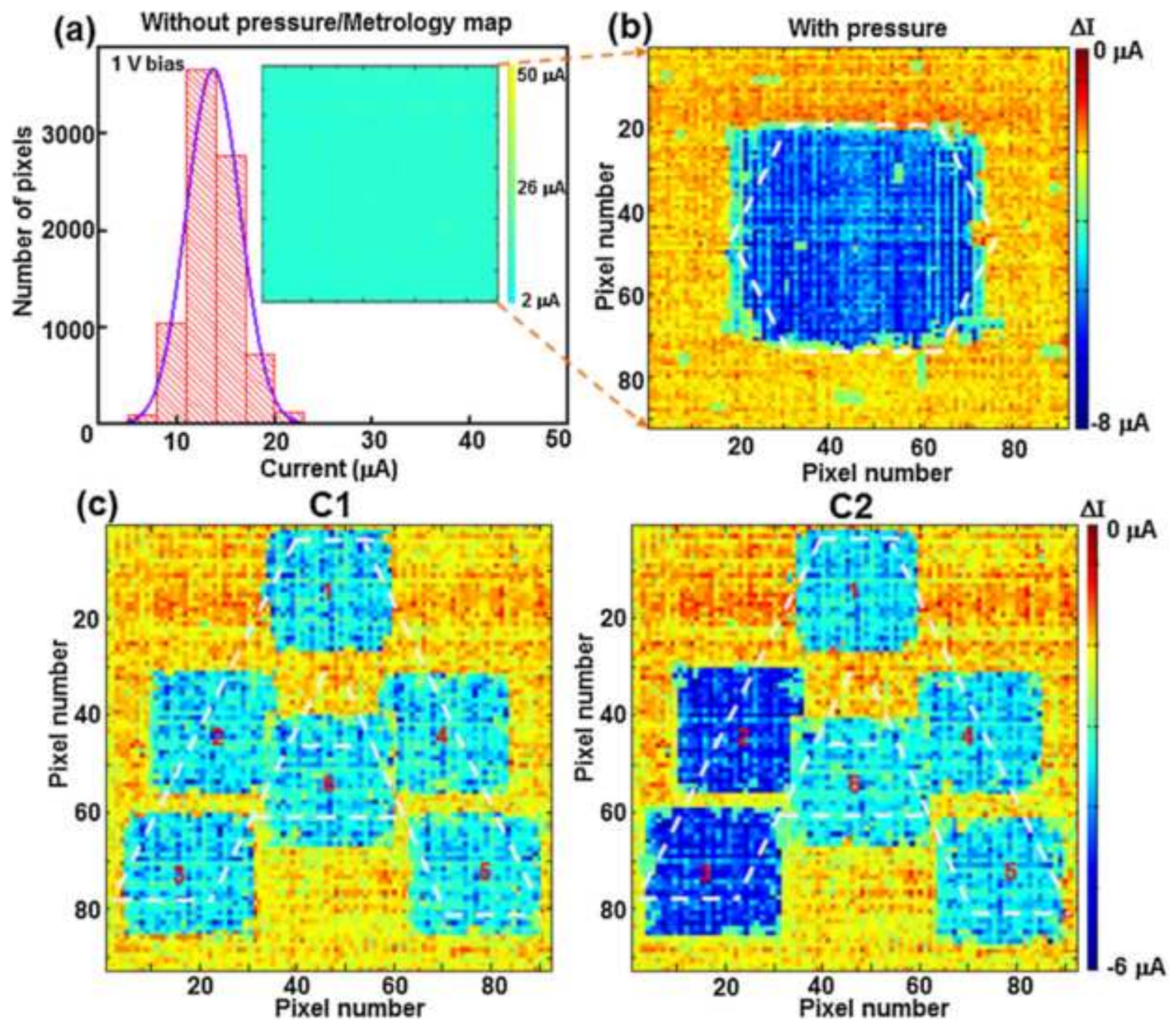
(a)
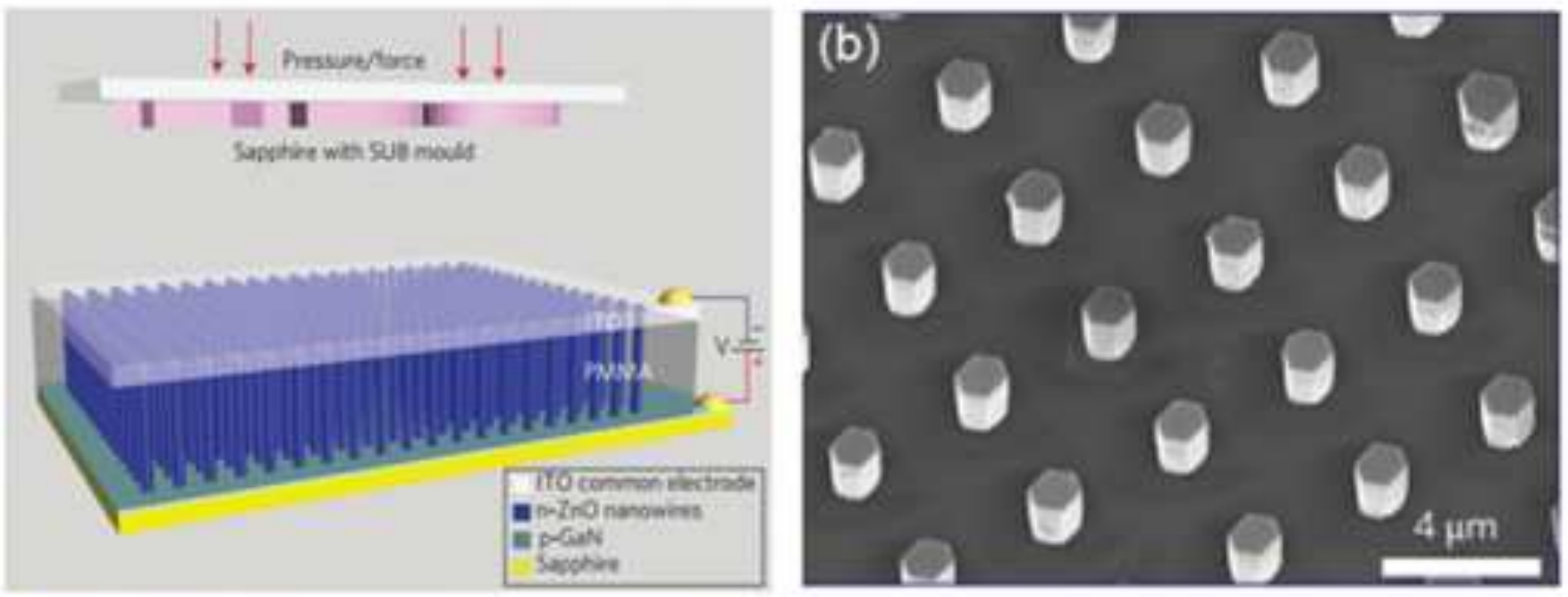

(c)
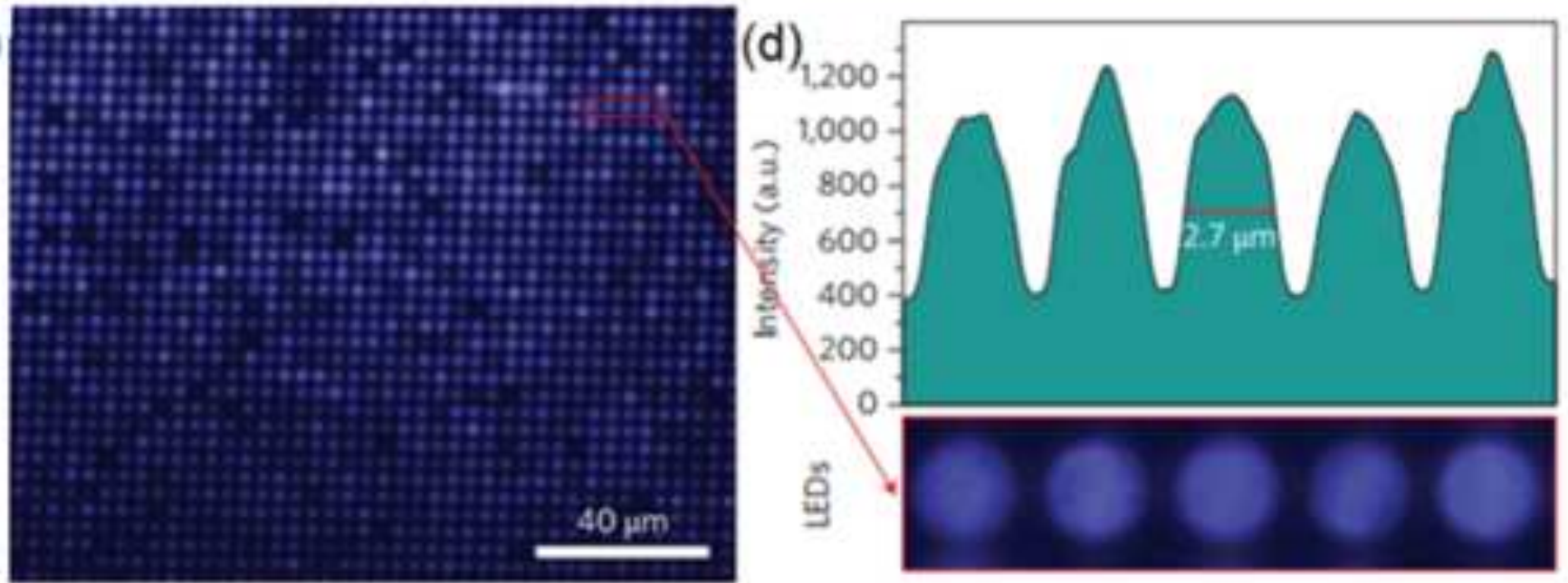

(e)

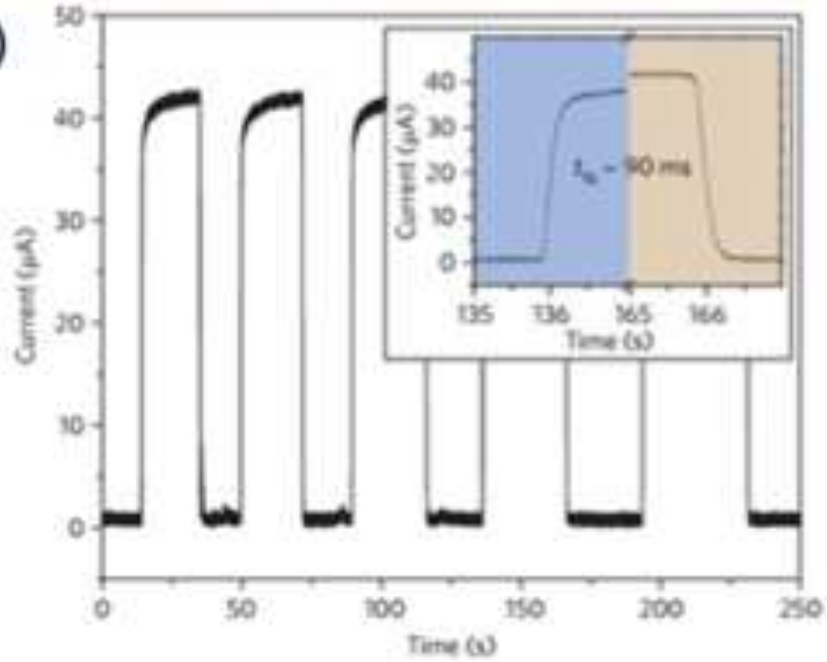

(f)

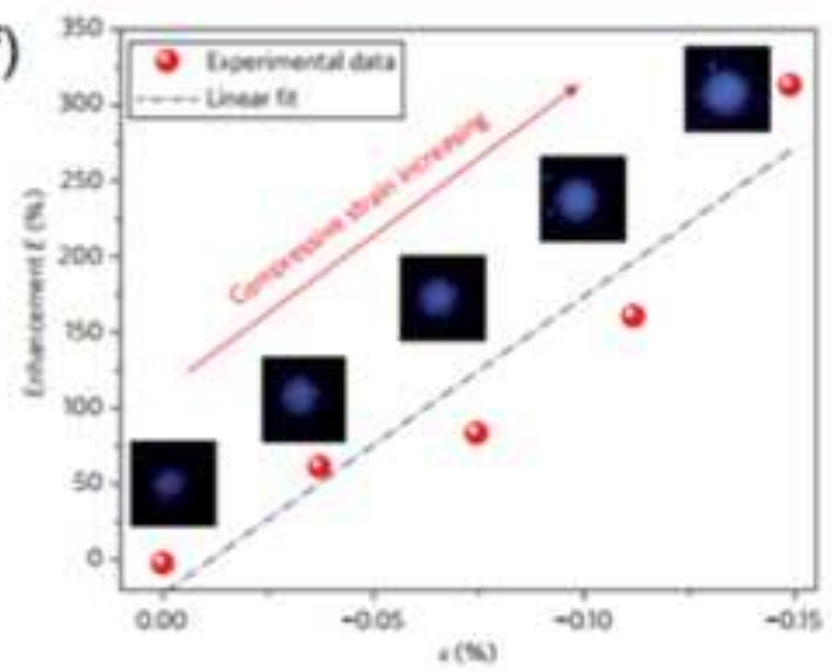



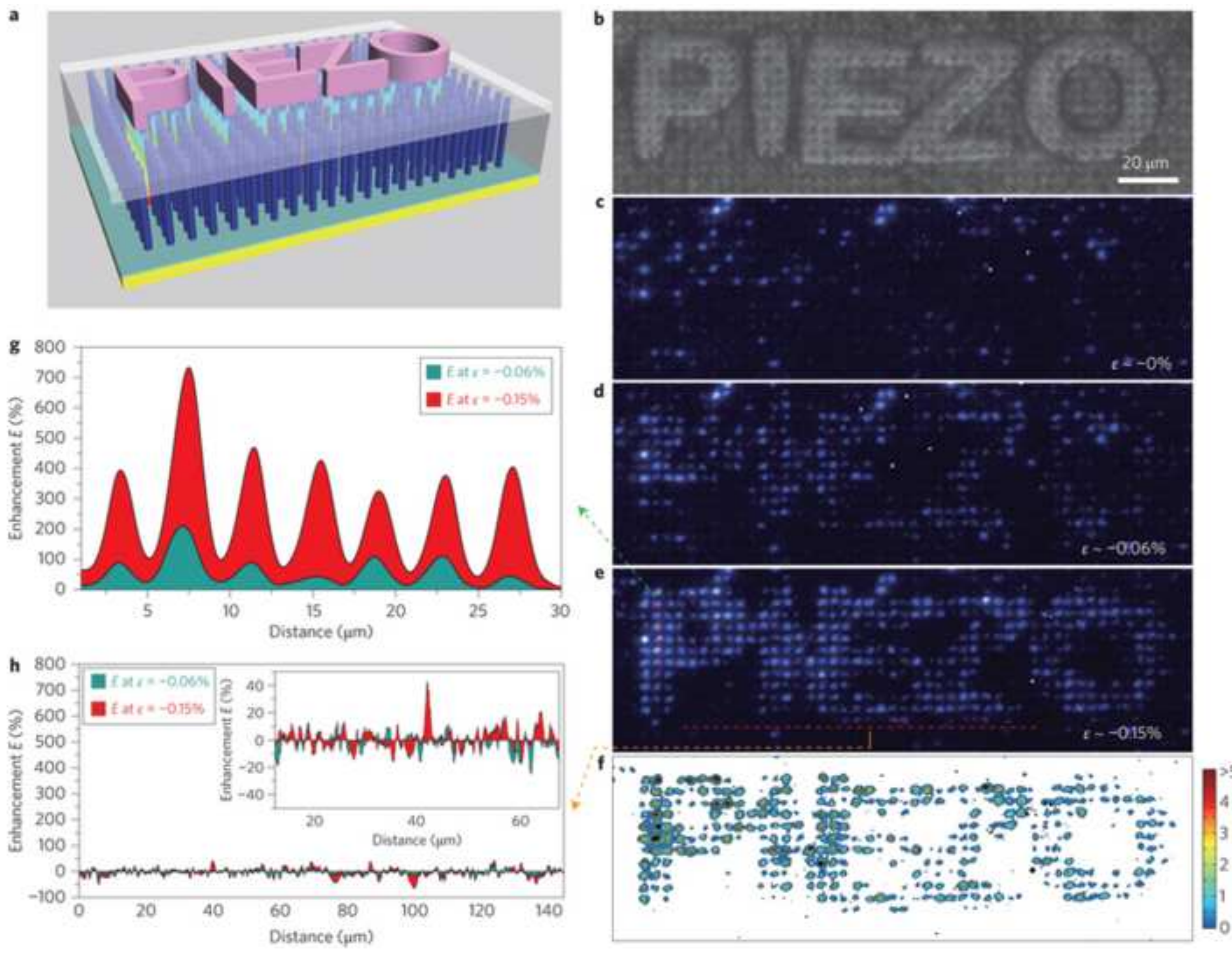


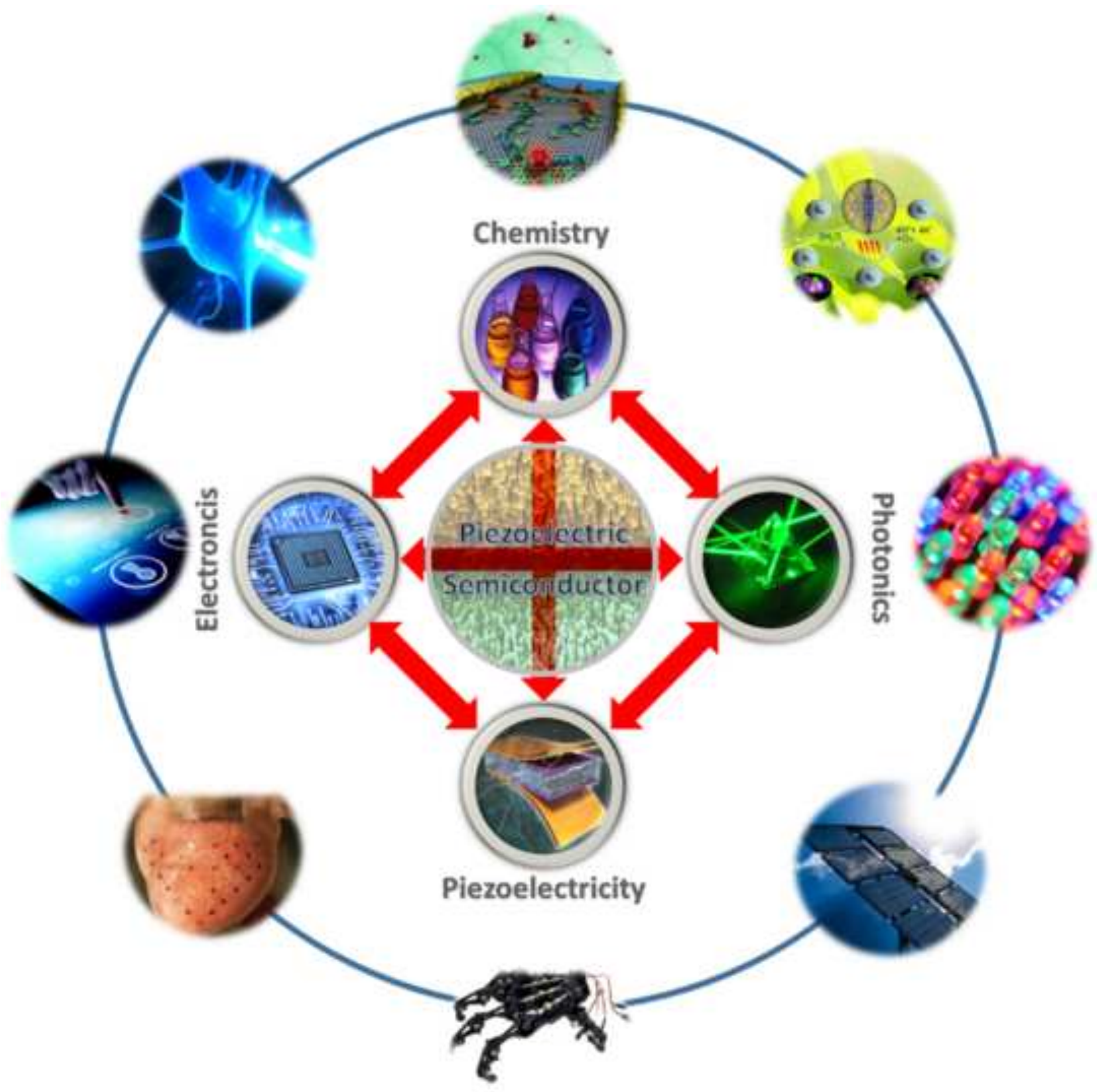




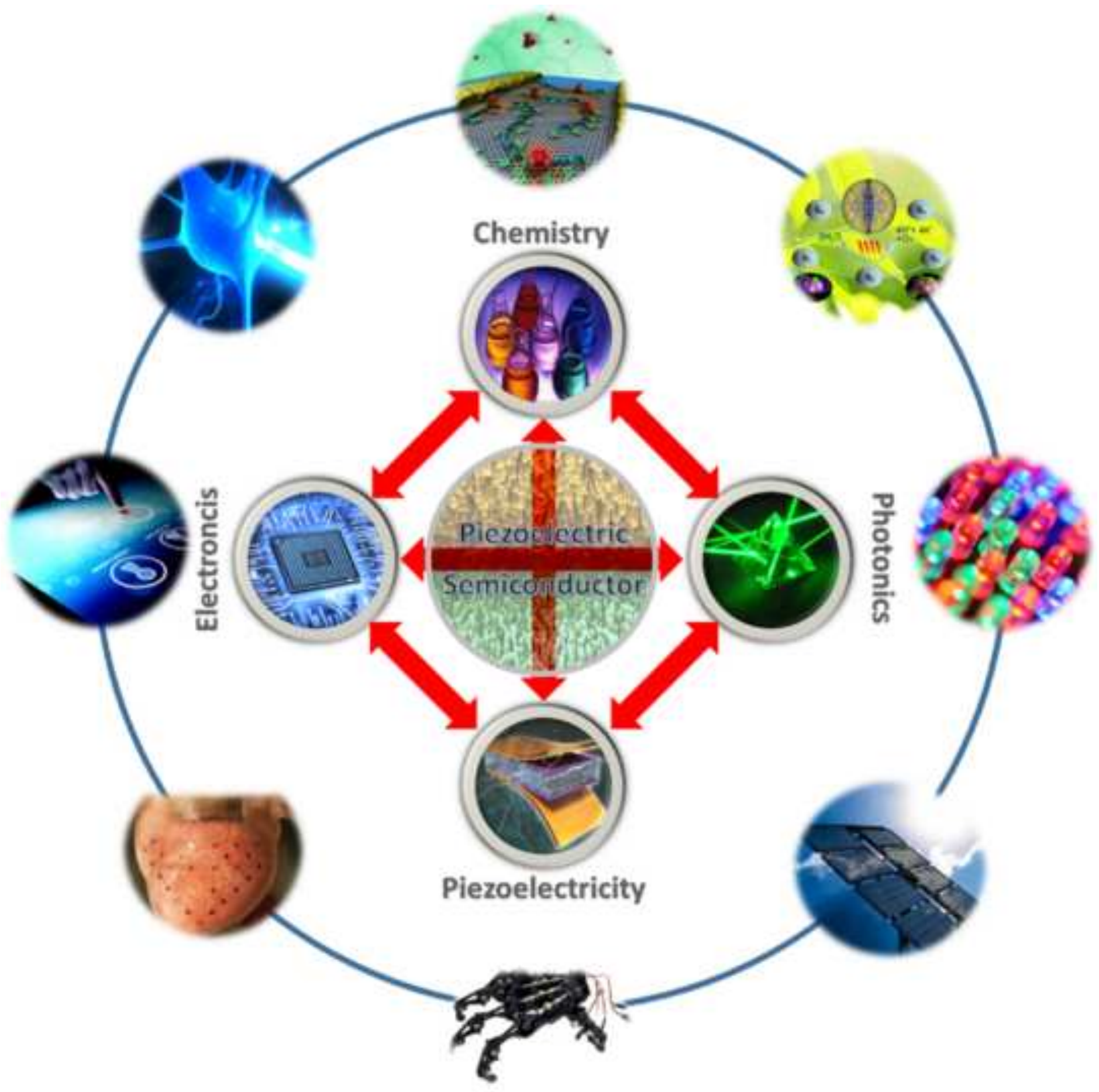

